Supporting Information

\title{
Visible-light Mediated di-Arylselenylative Cyclization of 1,6-Enynes
}

Hong Hou, ${ }^{a}$ Yue Sun, ${ }^{a}$ Yingjie Pan, ${ }^{a}$ Huaguang Yu, ${ }^{b}$ Ying Han, ${ }^{a}$ Yaocheng Shi, ${ }^{a}$ Chaoguo Yan, ${ }^{a}$ and Shaoqun Zhu*a

aSchool of Chemistry \& Chemical Engineering, Yangzhou University, Yangzhou, 225009, China

${ }^{b}$ Key Laboratory of Optoelectronic Chemical Materials and Devices, Ministry of Education, School of Chemical and Environmental Engineering, Jianghan University, Wuhan, 430056, China

1. X-ray Structure of $\mathbf{3 f}$ and experimental set-up information

2. ${ }^{1} \mathrm{H}$ NMR, ${ }^{13} \mathrm{C}\left\{{ }^{1} \mathrm{H}\right\}$ NMR and ${ }^{19} \mathrm{~F}\left\{{ }^{1} \mathrm{H}\right\}$ NMR spectra of products 


\section{Single-crystal X-ray data for compound $3 f$}

Single crystals of $\mathbf{3} \mathbf{f}$ were obtained by recrystallization from dichloromethane/n-hexane solution. The crystal structure was determined by standard crystallographic methods. A colorless block-shaped crystal $(0.03 \times$ $0.02 \times 0.01 \mathrm{~mm}^{3}$ ) was used for single-crystal X-ray diffraction. The data were collected at $273.15 \mathrm{~K}$ using a Bruker D8 QUEST X-ray diffractometer with graphite-monochromated Mo-K $\alpha$ radiation $(\lambda=0.71073 \AA)$. The structure was solved by Direct Methods and refined by full-matrix least-squares techniques on F2 with anisotropic displacement parameters for all atoms using SHELX-2014 ${ }^{[1]}$. All the processes were performed within Olex2. The final refinements included anisotropic displacements parameters for all atoms and a secondary extinction correction. The crystallographic parameter data is listed in Table S1.

Crystallographic data for $\mathbf{3 f}$ have been deposited with the Cambridge Crystallographic Data Center as CCDC: 2023465. And the crystal data and details of the data collection are given in Table S1.

Table S1. Crystal data of $\mathbf{3 f}$.

\begin{tabular}{|c|c|c|}
\hline Empirical formula & \multicolumn{2}{|l|}{$\mathrm{C}_{30} \mathrm{H}_{33} \mathrm{NO}_{2} \mathrm{SSe}_{2}$} \\
\hline $\mathrm{CCDC}$ & \multicolumn{2}{|l|}{2023465} \\
\hline Formula weight & \multicolumn{2}{|l|}{629.55} \\
\hline Temperature $(\mathrm{K})$ & \multicolumn{2}{|l|}{273.15} \\
\hline Wavelength $(\AA)$ & \multicolumn{2}{|l|}{0.71073} \\
\hline Crystal system & \multicolumn{2}{|l|}{ triclinic } \\
\hline space group & \multicolumn{2}{|l|}{$\mathrm{P}-1$} \\
\hline \multirow{3}{*}{ Unit cell dimensions } & $\mathrm{a}=10.3217(6) \AA$ & $\alpha=90.960(2)^{\circ}$ \\
\hline & $\mathrm{b}=15.6829(10) \AA$ & $\beta=103.262(2)^{\circ}$ \\
\hline & $\mathrm{c}=18.1401(10) \AA$ & $\gamma=92.248(2)^{\circ}$ \\
\hline Volume $\left(\AA^{3}\right)$ & \multicolumn{2}{|l|}{$2854.9(3)$} \\
\hline Z & \multicolumn{2}{|l|}{4} \\
\hline Calculated density $\left(\mathrm{g} \cdot \mathrm{cm}^{-3}\right)$ & \multicolumn{2}{|l|}{1.465} \\
\hline Absorption coefficient $\left(\mathrm{mm}^{-1}\right)$ & \multicolumn{2}{|l|}{2.690} \\
\hline $\mathrm{F}(000)$ & \multicolumn{2}{|l|}{1280.0} \\
\hline Crystal size $(\mathrm{mm})$ & \multicolumn{2}{|l|}{$0.23 \times 0.22 \times 0.21$} \\
\hline$\theta$ range for data collection & \multicolumn{2}{|l|}{4.058 to 55.09} \\
\hline$h, k, l$ ranges & \multicolumn{2}{|c|}{$-13<=\mathrm{h}<=13,-20<=\mathrm{k}<=20,-23<=1<=23$} \\
\hline Reflections collected & \multicolumn{2}{|l|}{45094} \\
\hline Independent reflections & \multicolumn{2}{|c|}{$13068[\mathrm{R}(\mathrm{int})=0.0704]$} \\
\hline Completeness & \multicolumn{2}{|l|}{$99.1 \%$} \\
\hline Absorption correction & \multicolumn{2}{|l|}{ multi-scan } \\
\hline Data / restraints / parameters & \multicolumn{2}{|l|}{$13068 / 1 / 651$} \\
\hline Goodness-of-fit on $F^{2}$ & \multicolumn{2}{|l|}{1.010} \\
\hline Final $R$ indices $[\mathrm{I}>2 \sigma(\mathrm{I})]$ & \multicolumn{2}{|c|}{$\mathrm{R}_{1}=0.0560, \mathrm{wR}_{2}=0.0990$} \\
\hline$R$ indices (all data) & \multicolumn{2}{|c|}{$\mathrm{R}_{1}=0.1309, \mathrm{wR}_{2}=0.1181$} \\
\hline Largest diff. peak and hole & \multicolumn{2}{|c|}{1.01 and $-0.78 \mathrm{e} \cdot \AA^{-3}$} \\
\hline
\end{tabular}


Figure S1. Crystal Structure of $\mathbf{3 f}$ with thermal ellipsoids drawn at the $30 \%$ probability level.
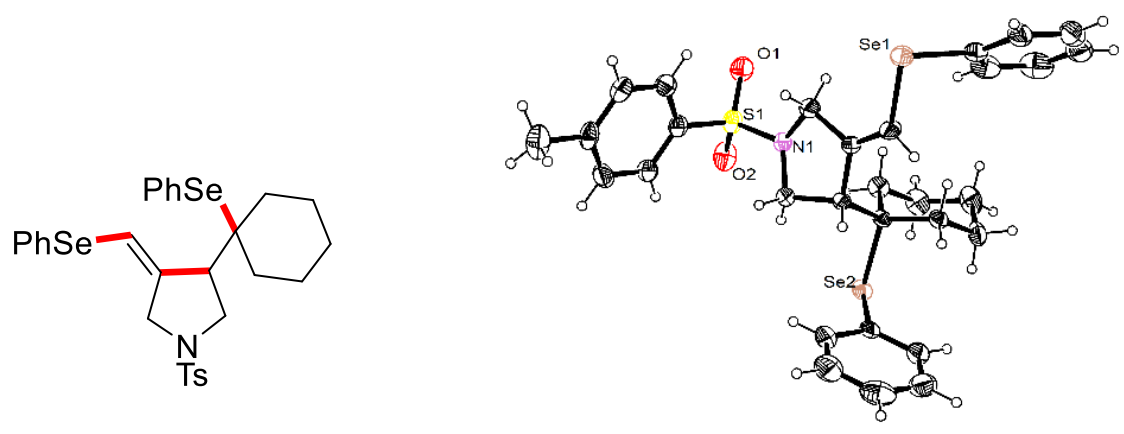

CCDC: 2023465

\section{References:}

[1] Dolomanov, O. V.; Bourhis, L. J.; Gildea, R. J.; Howard, J. A. K.; Puschmann, H. OLEX2: A Complete Structure Solution, Refinement and Analysis Program. J. Appl. Crystallogr. 2009, 42, 339-341.

Figure S2. Light Source, Material of the Irradiation Vessel and Photo of the experimental set-up.

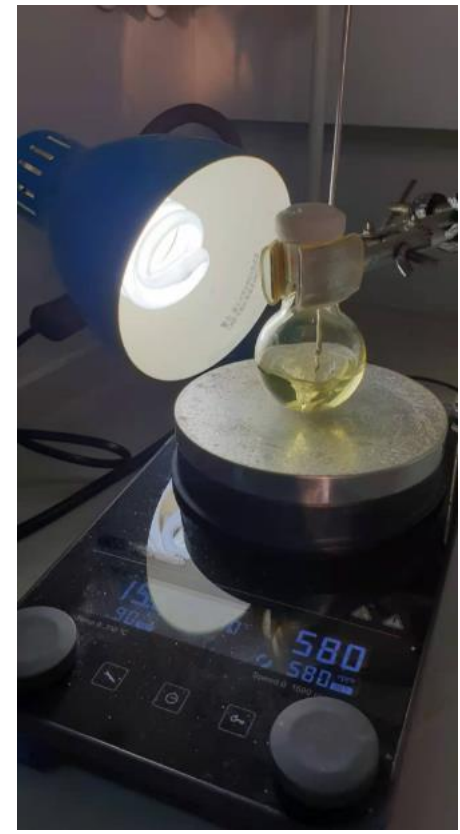

Gram-scale reaction

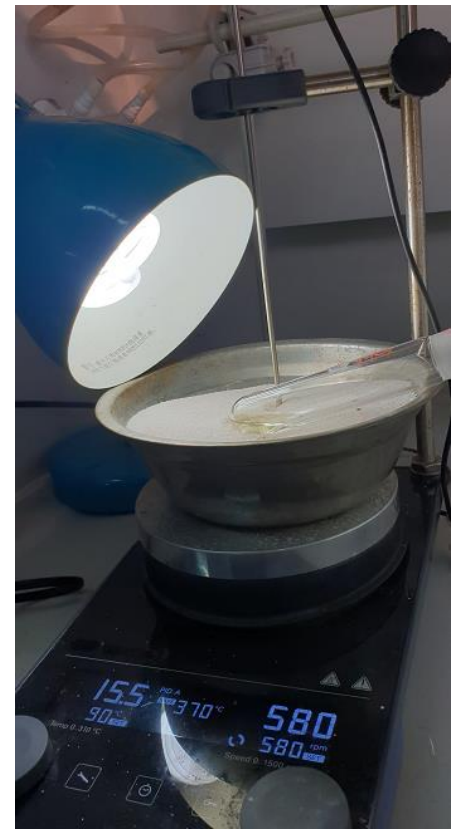

Small scale reaction

The light source used for photochemical experiments was a household $23 \mathrm{~W}$ fluorescent bulb (bulb Rating 220V 50 Hz), purchased from JD.COM;

https://item.jd.com/25727786197.html\#crumb-wrap

Manufacturer: Philips. China; Model: YPZ220V23W50Hz/S-RR-N;

Broadband source: $\lambda=405 \sim 577 \mathrm{~nm}$;

Material of the irradiation vessel: Borosilicate reaction tube $(10 \mathrm{~mL})$ or Borosilicate flask $(25 \mathrm{~mL})$;

Distance from the light source to the irradiation vessel: $5.0 \mathrm{~cm}$ (Not use any filters) 


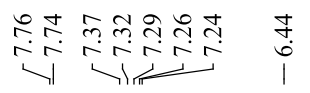

2. ${ }^{1} \mathrm{H}$ NMR, ${ }^{13} \mathrm{C}\left\{{ }^{1} \mathrm{H}\right\}$ NMR and ${ }^{19} \mathrm{~F}\left\{{ }^{1} \mathrm{H}\right\}$ NMR spectra of products
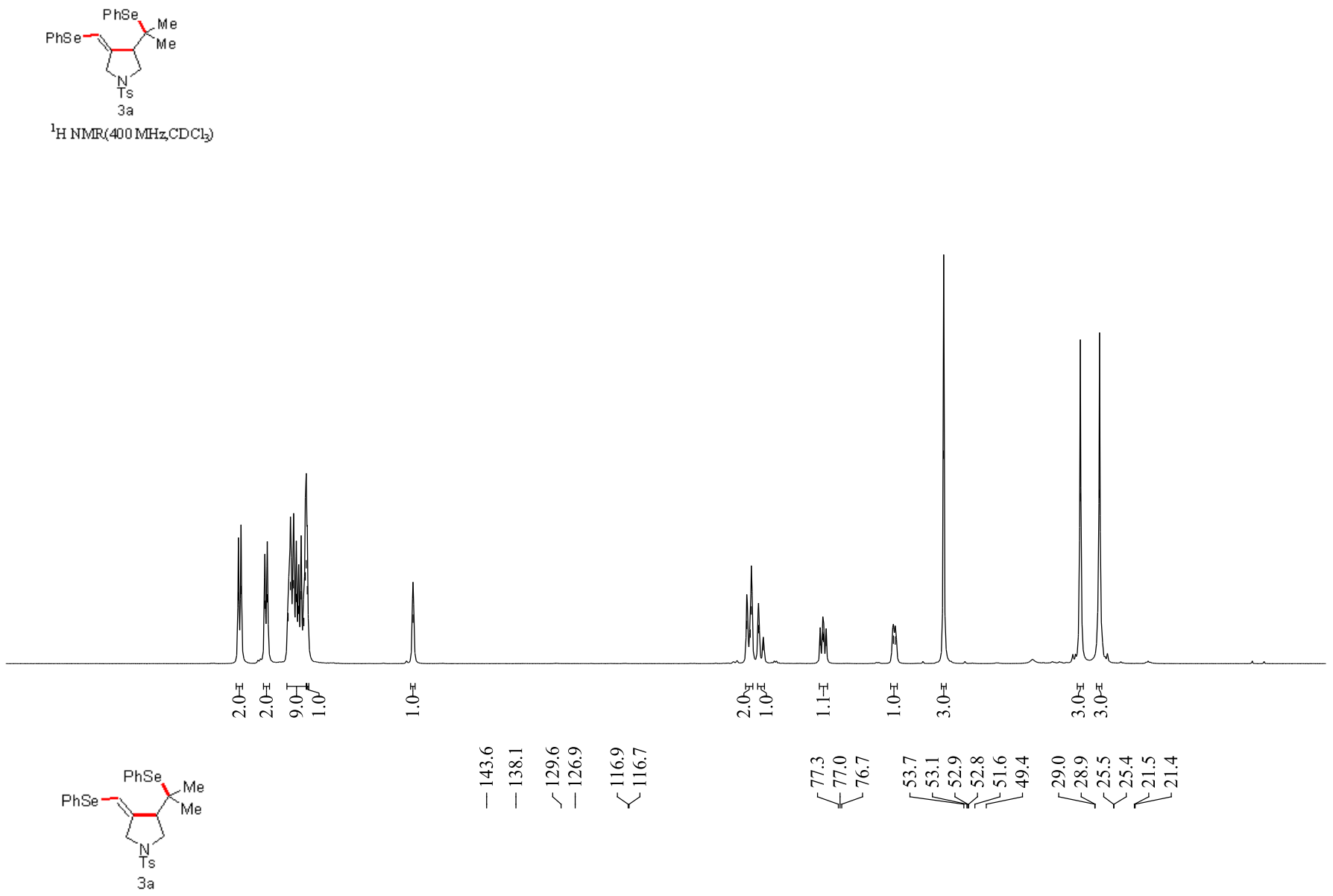

${ }^{13} \mathrm{C}\left\{{ }^{1} \mathrm{H}\right\}$ N MR $\left(101 \mathrm{MHz}, \mathrm{CDCl}_{3}\right)$

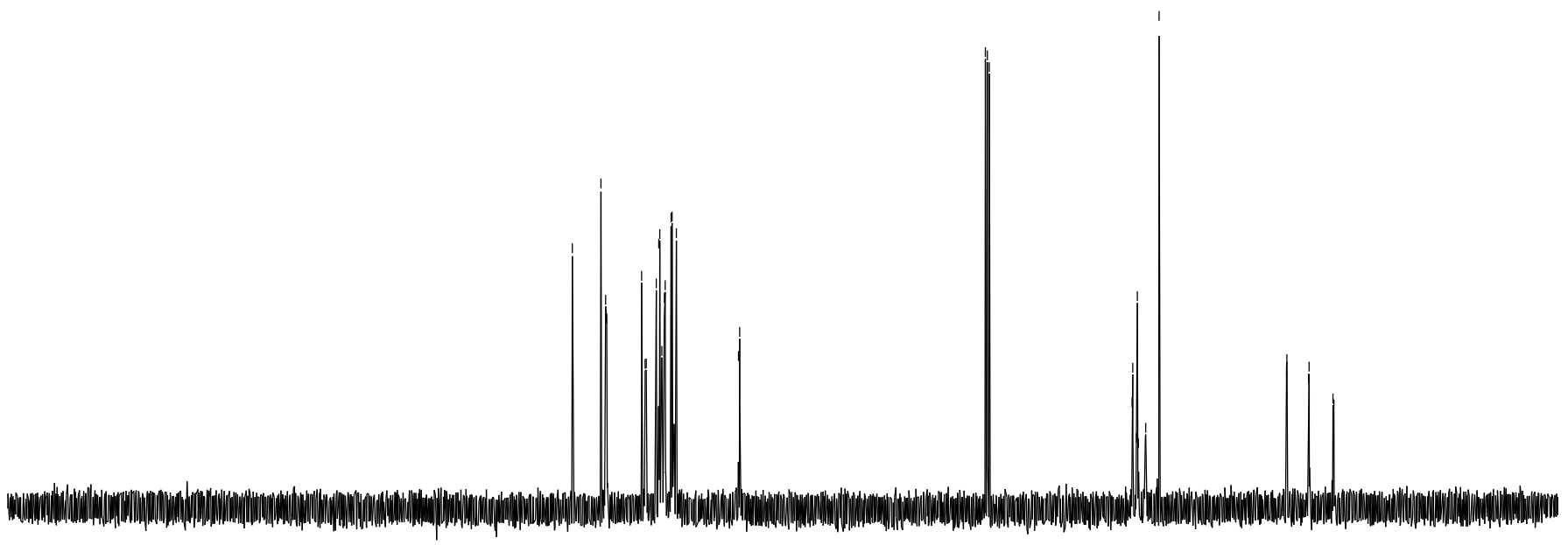




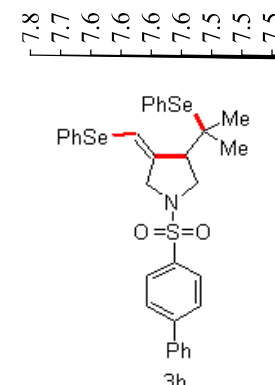

${ }^{1} \mathrm{H} \mathrm{NMR}\left(400 \mathrm{MHz}, \mathrm{CDCl}_{3}\right)$

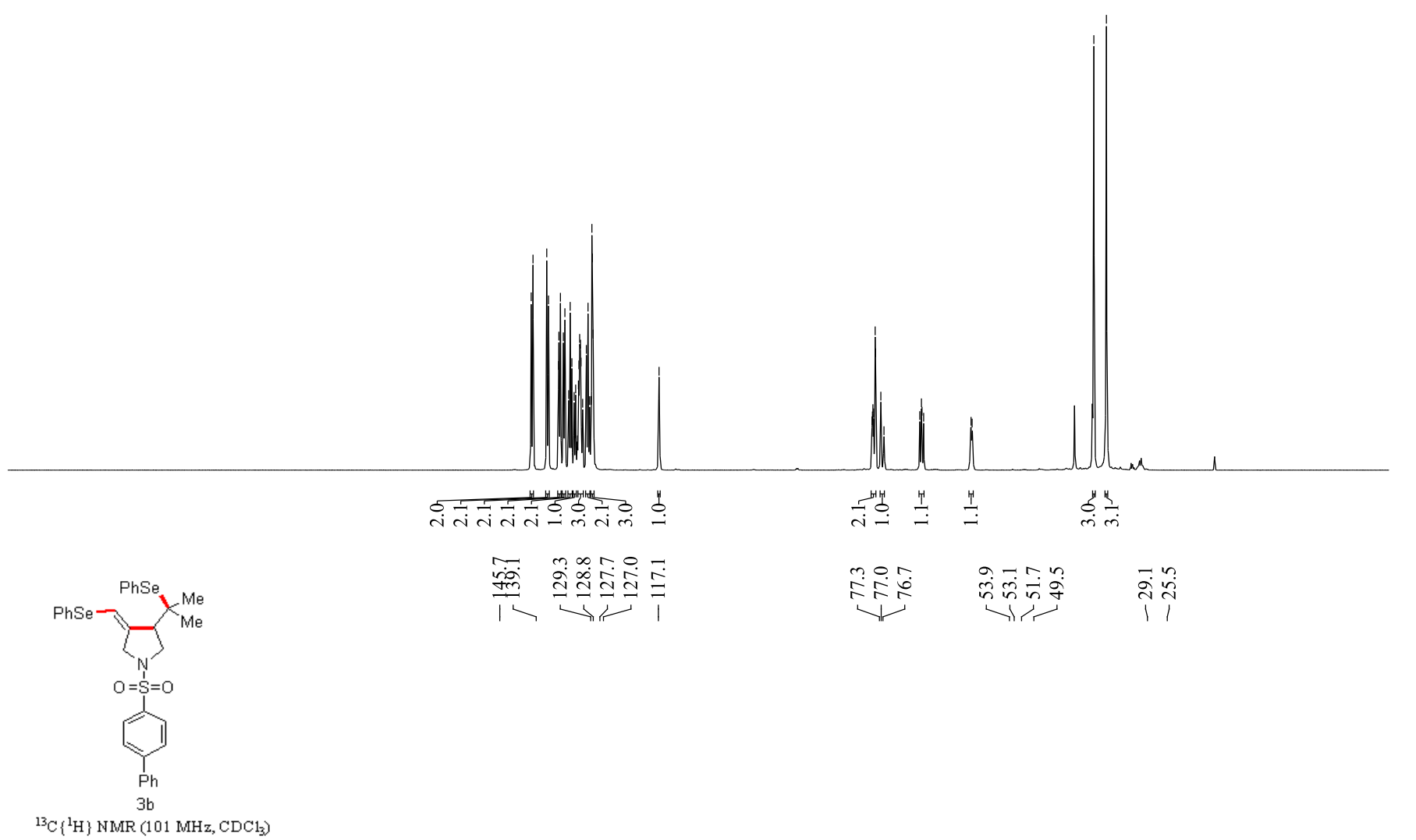

${ }^{13} \mathrm{C}\left\{{ }^{1} \mathrm{H}\right\}$ NMR $\left(101 \mathrm{MHz}, \mathrm{CDCl}_{3}\right)$ 


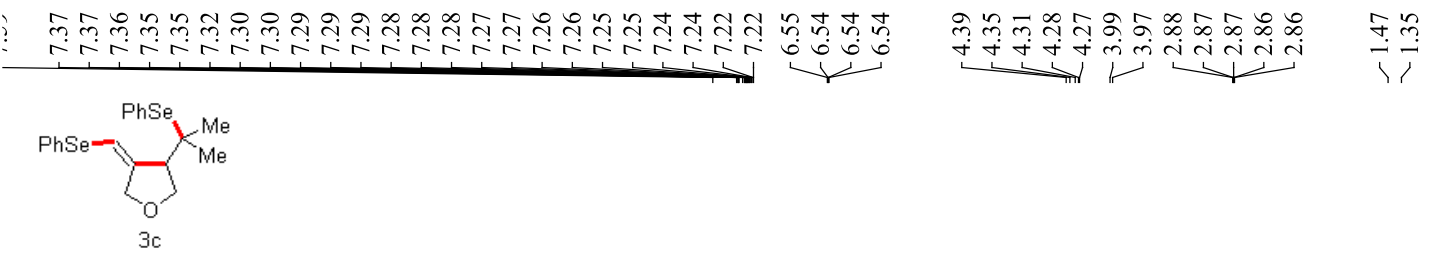

${ }^{1} \mathrm{H} \mathrm{NMR}\left(400 \mathrm{MHz}_{\mathrm{z}} \mathrm{CDCl}_{3}\right)$
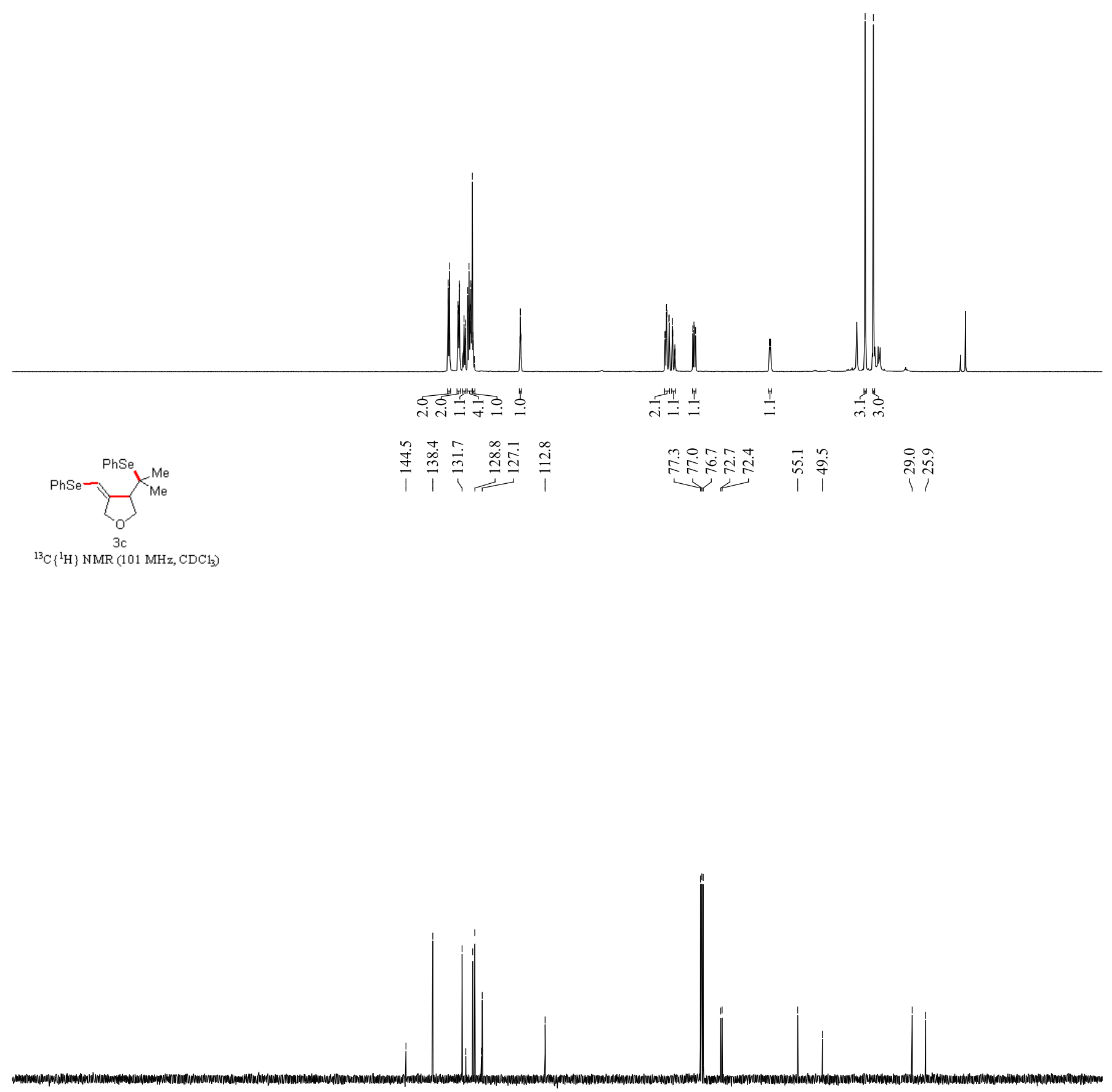


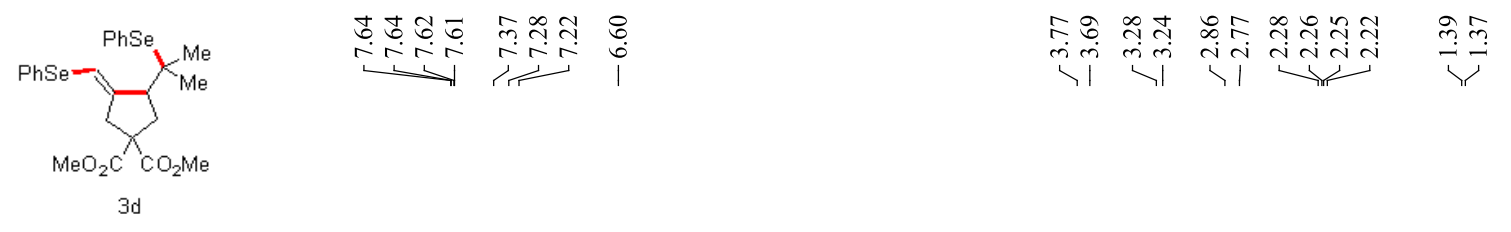

${ }^{1} \mathrm{H} \mathrm{NMR}\left(400 \mathrm{MHz}, \mathrm{CDCl}_{3}\right)$

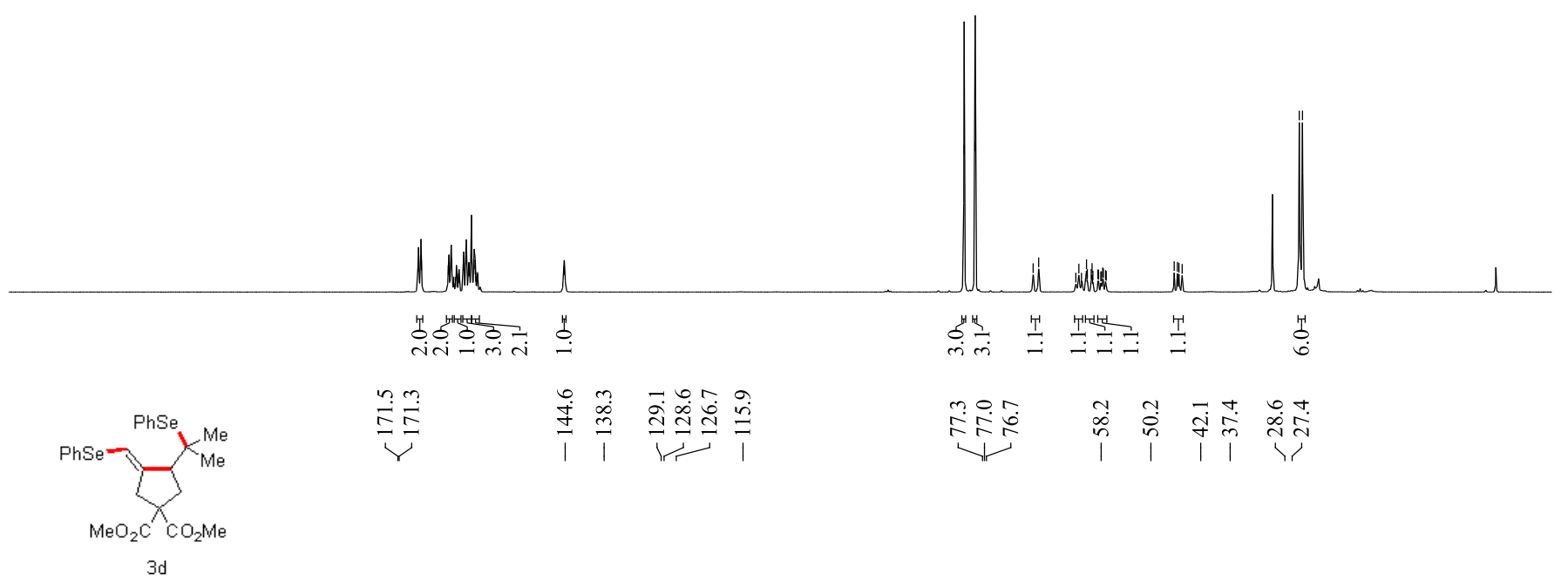

${ }^{13} \mathrm{C}\left\{{ }^{1} \mathrm{H}\right\}$ NMR $\left(101 \mathrm{MHz}, \mathrm{CDCl}_{3}\right)$

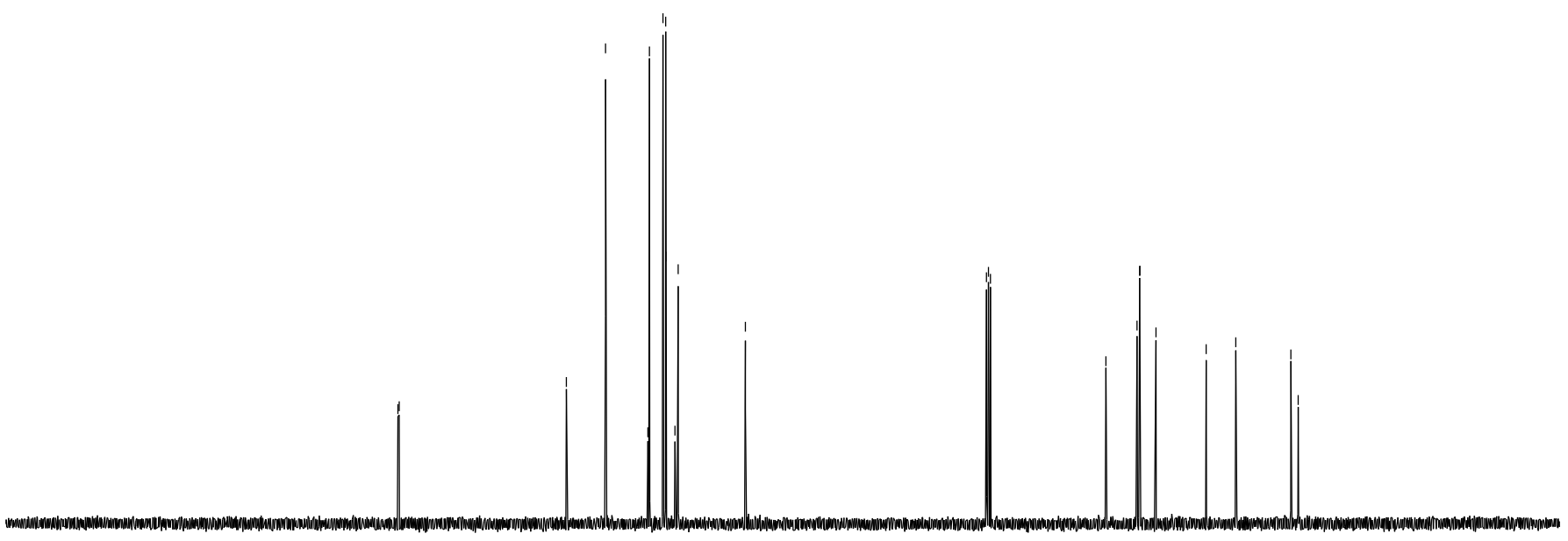



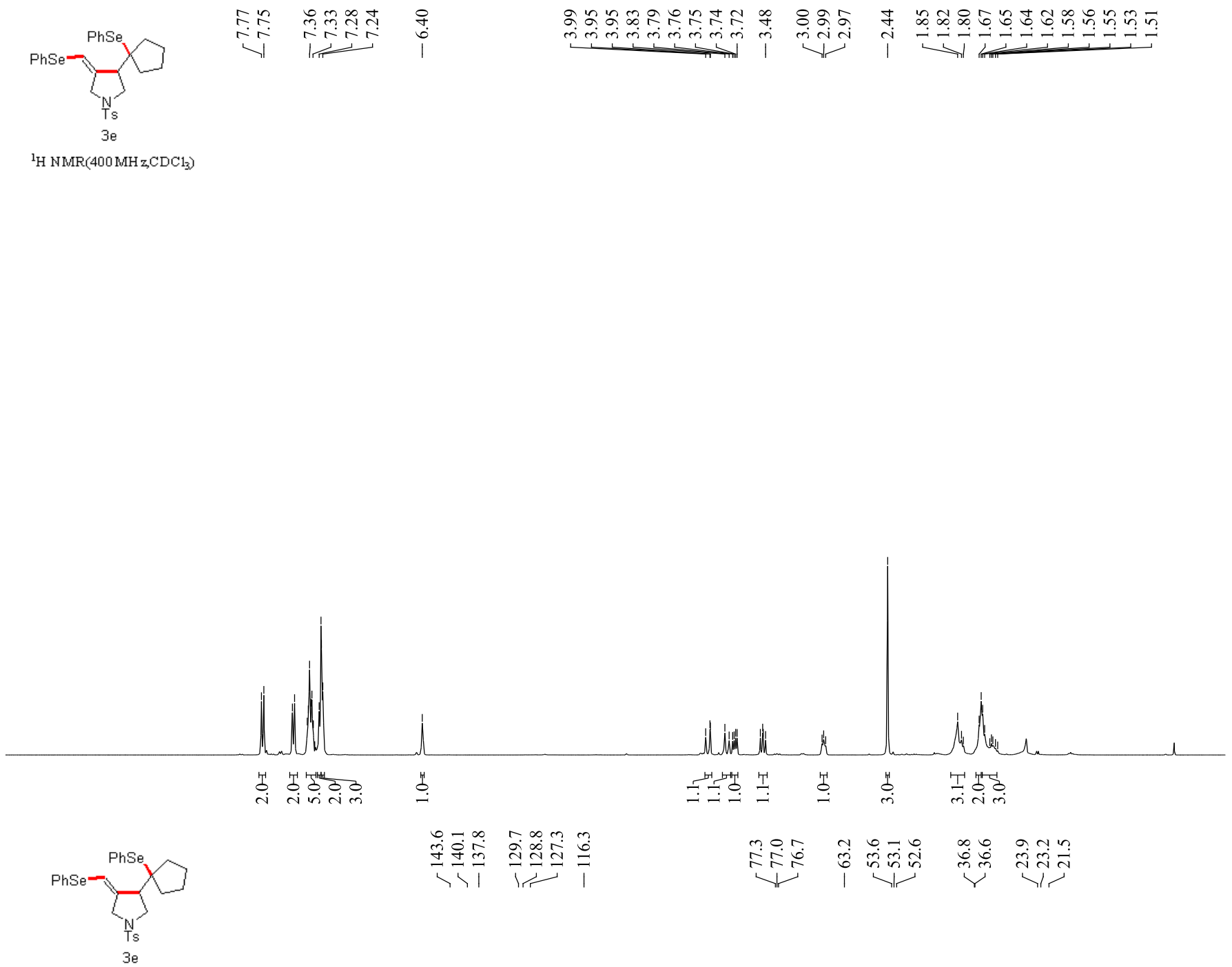

${ }^{13} \mathrm{C}\left\{{ }^{1} \mathrm{H}\right\}$ NMR $\left(101 \mathrm{MHz}, \mathrm{CDCl}_{3}\right)$ 

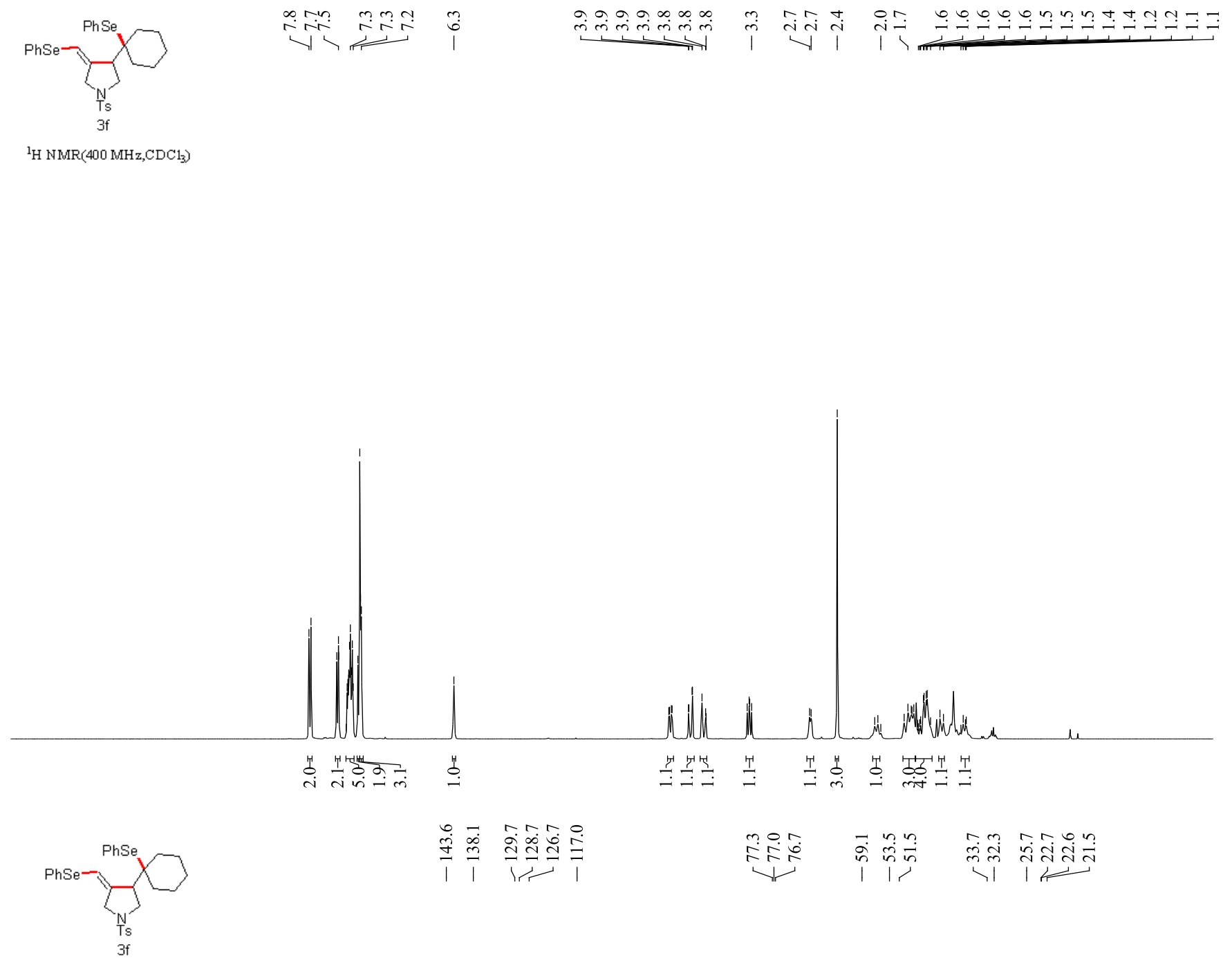

${ }^{13} \mathrm{C}\left\{{ }^{1} \mathrm{H}\right\} \mathrm{NMR}\left(101 \mathrm{MHz}, \mathrm{CDCl}_{3}\right)$ 

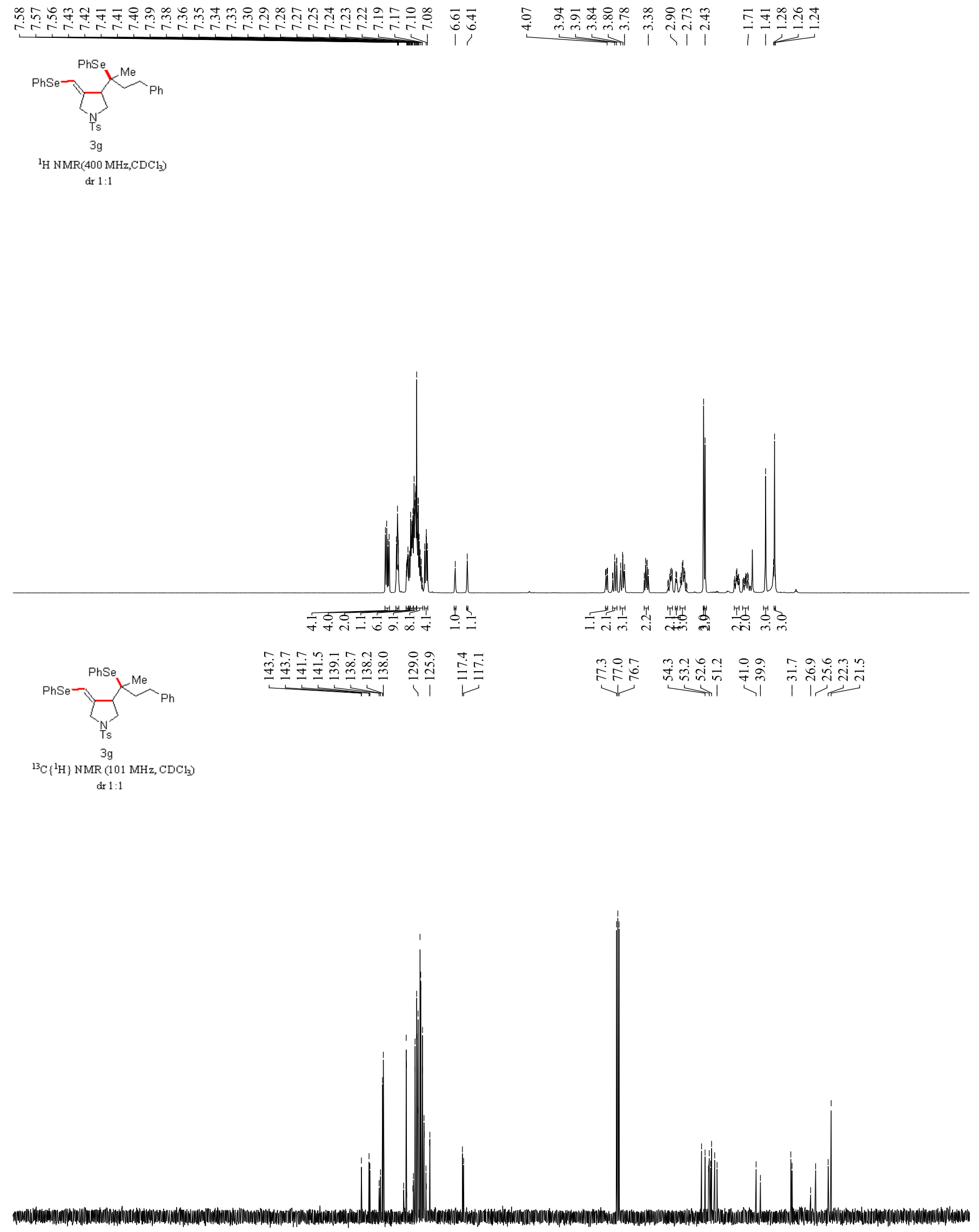

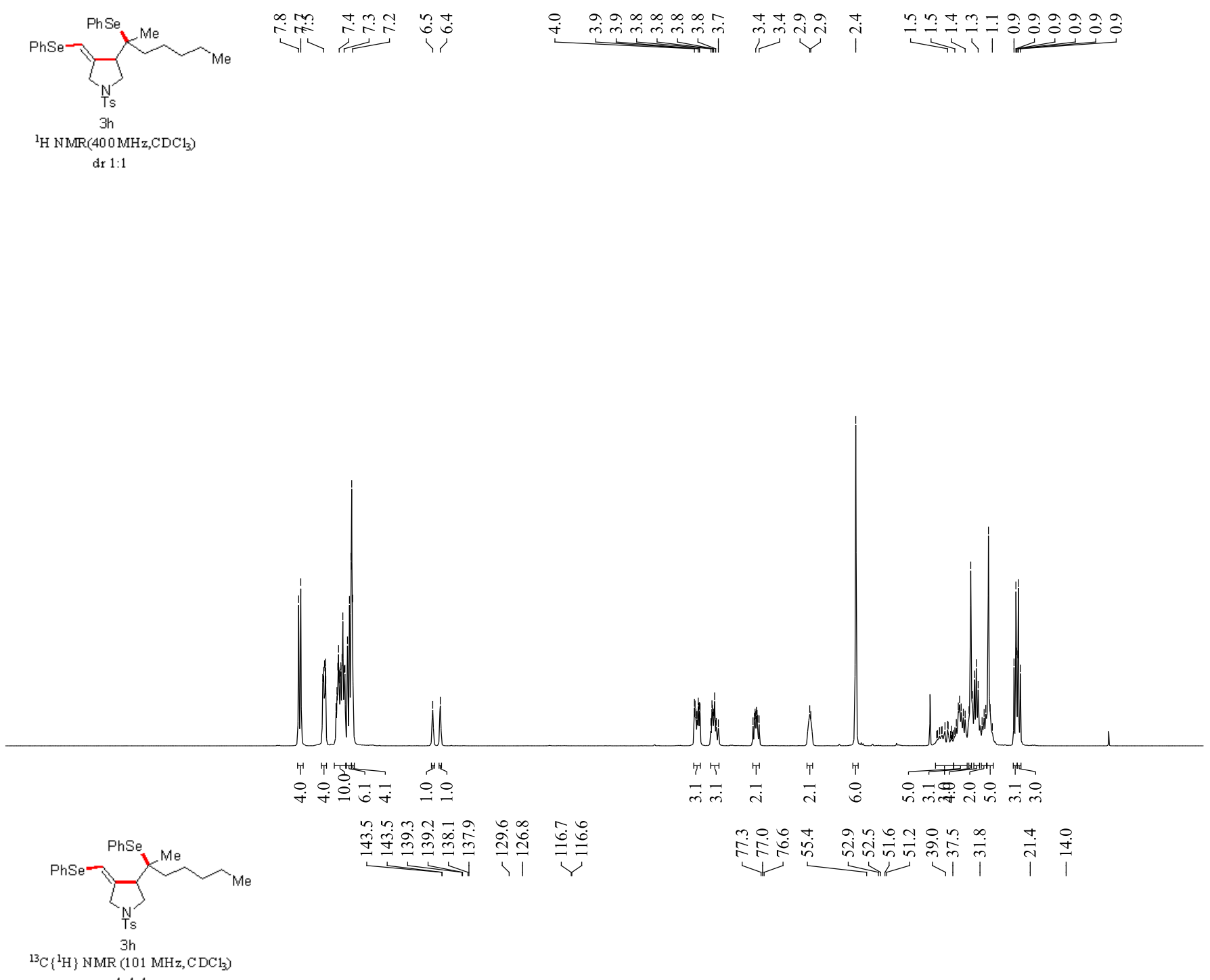

${ }^{13} \mathrm{C}\left\{{ }^{1} \mathrm{H}\right\} \operatorname{NMR}\left(101 \mathrm{MHz}, \mathrm{CDCl} 1_{3}\right)$

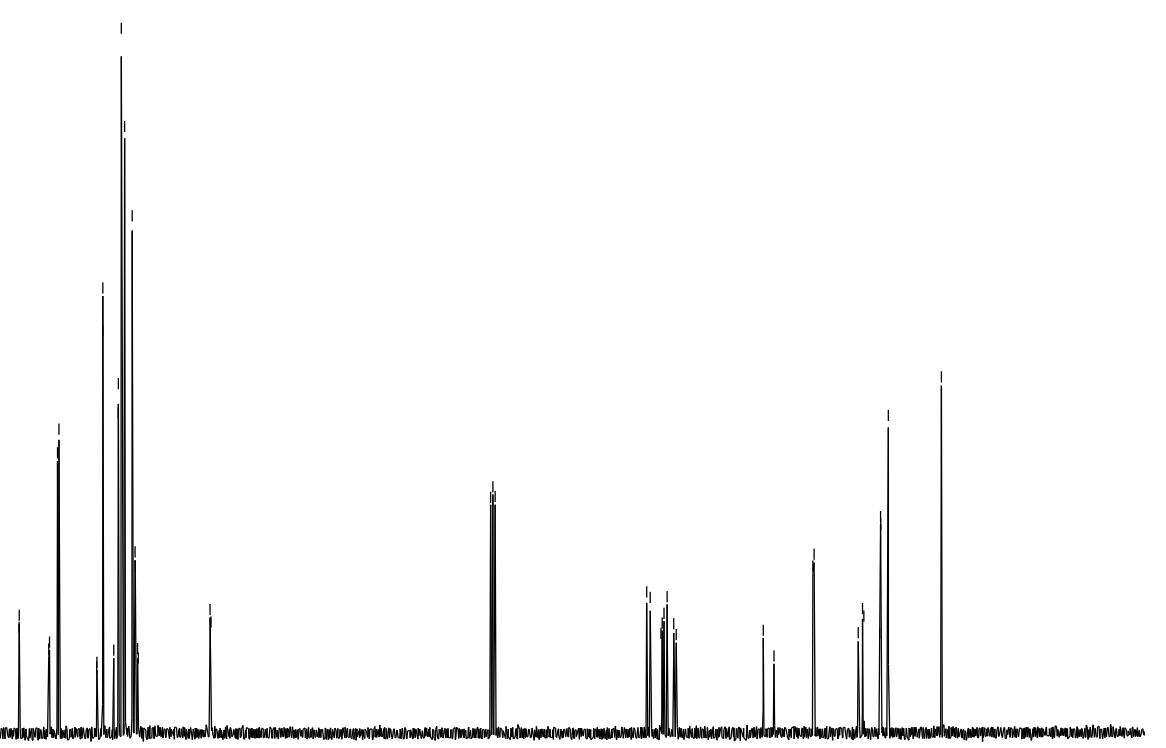




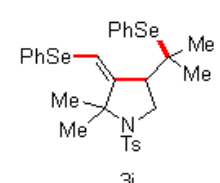

${ }^{1} \mathrm{H} \mathrm{NMR}\left(400 \mathrm{MHz}, \mathrm{CDCl}_{3}\right)$

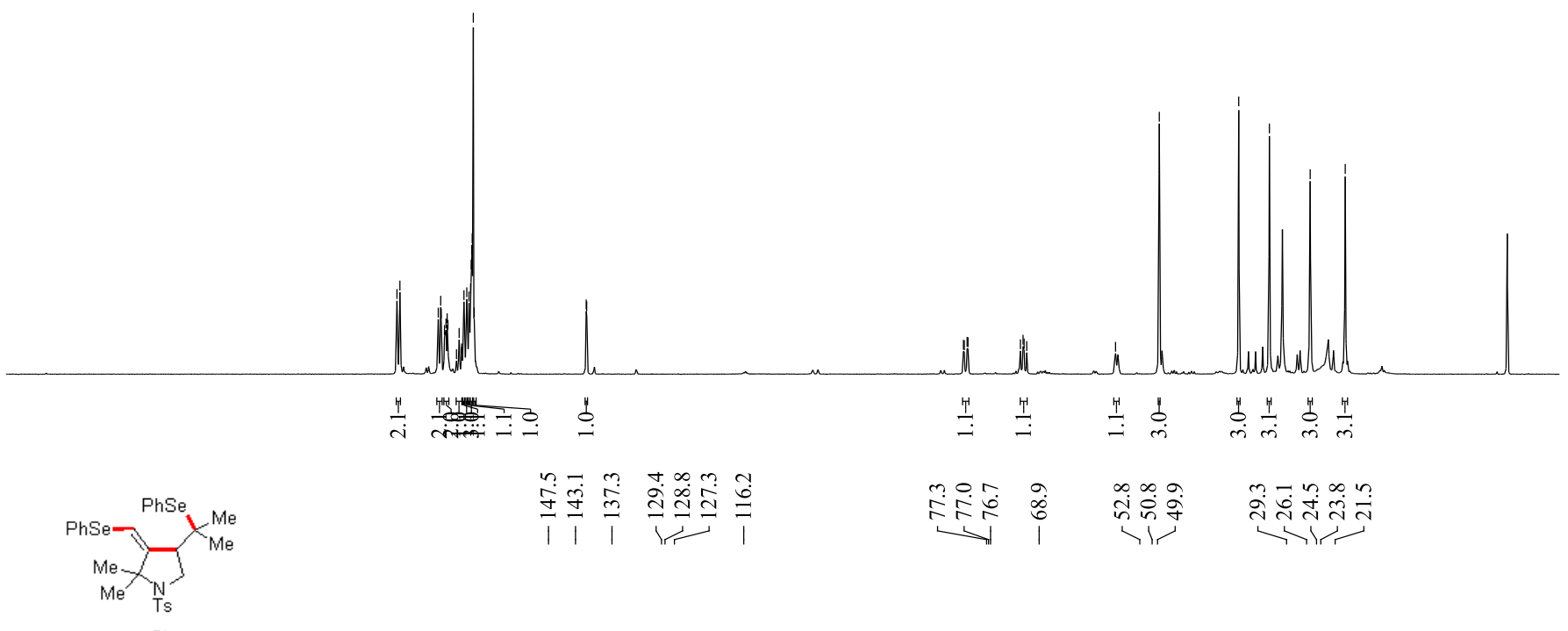

${ }^{13} \mathrm{C}\left\{{ }^{1} \mathrm{H}\right\} \mathrm{NMR}\left(101 \mathrm{MHz}, \mathrm{CDCl}_{3}\right)$

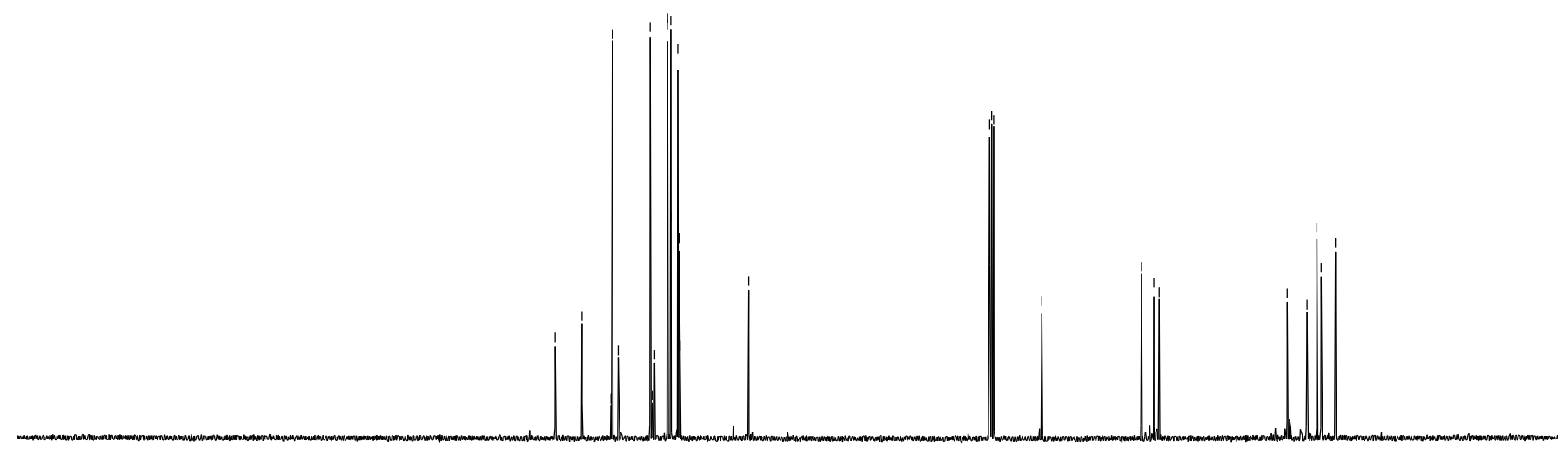



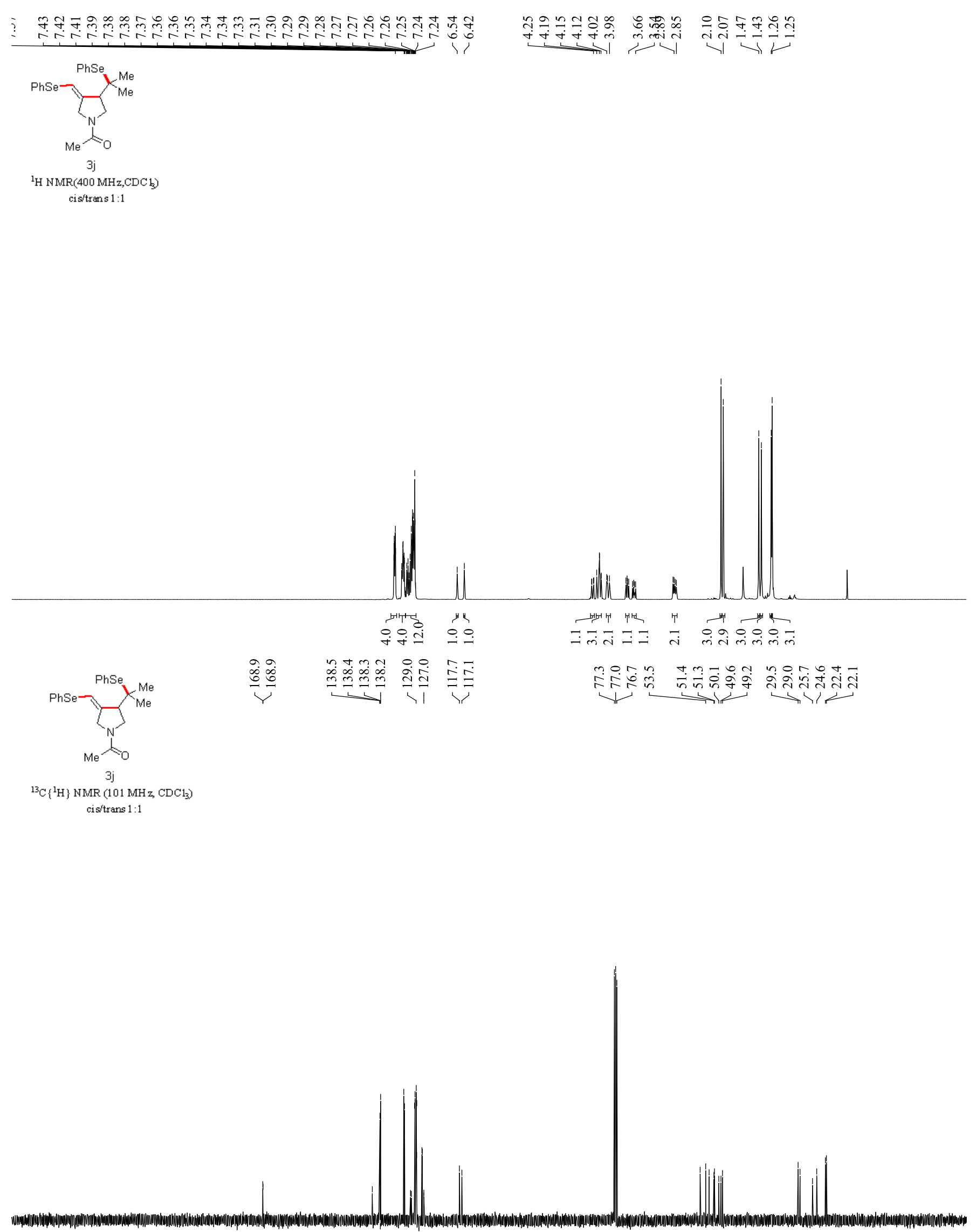

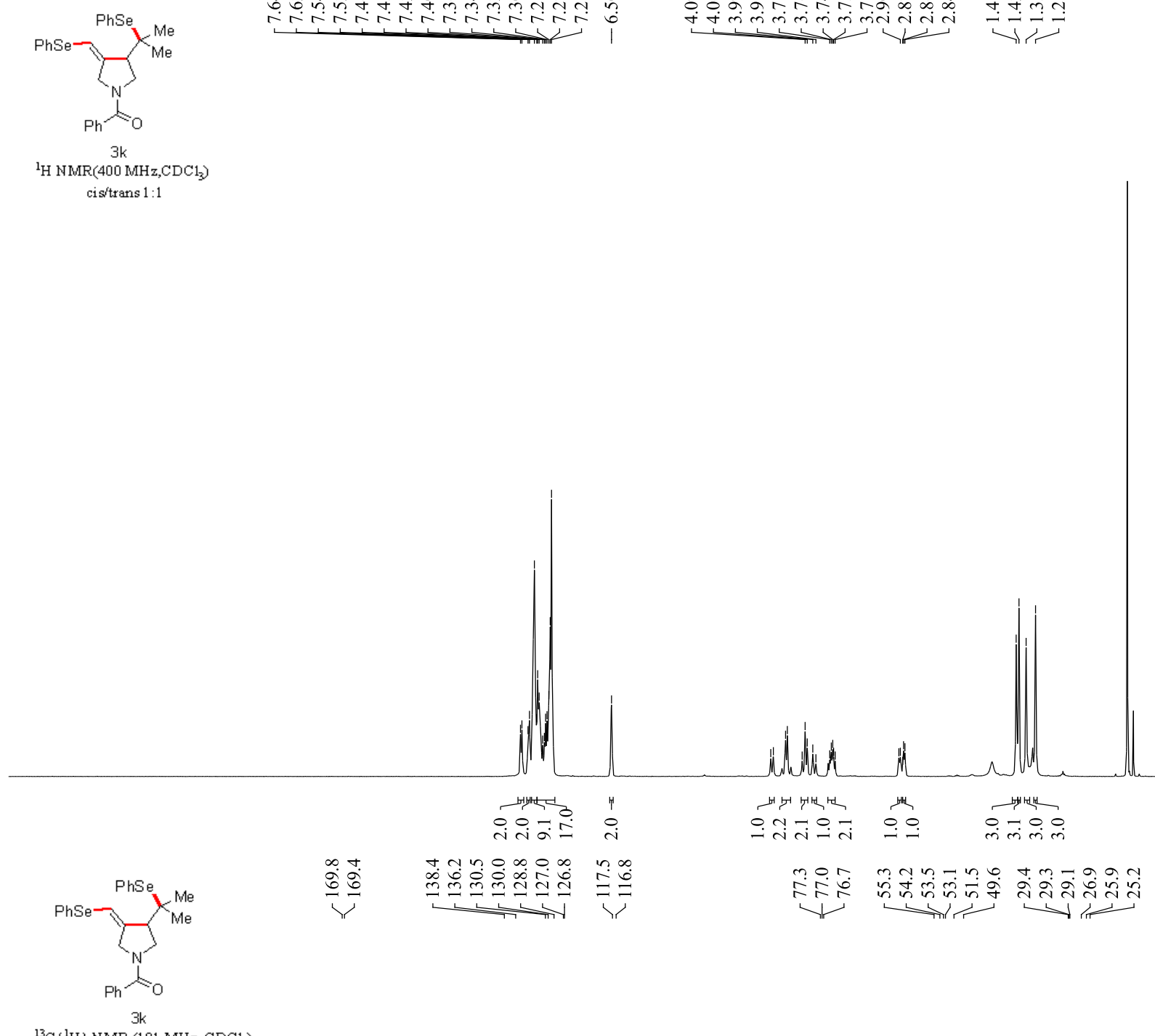

${ }^{13} \mathrm{C}\left\{{ }^{1} \mathrm{H}\right\} \mathrm{NMR}\left(101 \mathrm{MHz}, \mathrm{CDCl}_{3}\right)$

cisittans $1: 1$

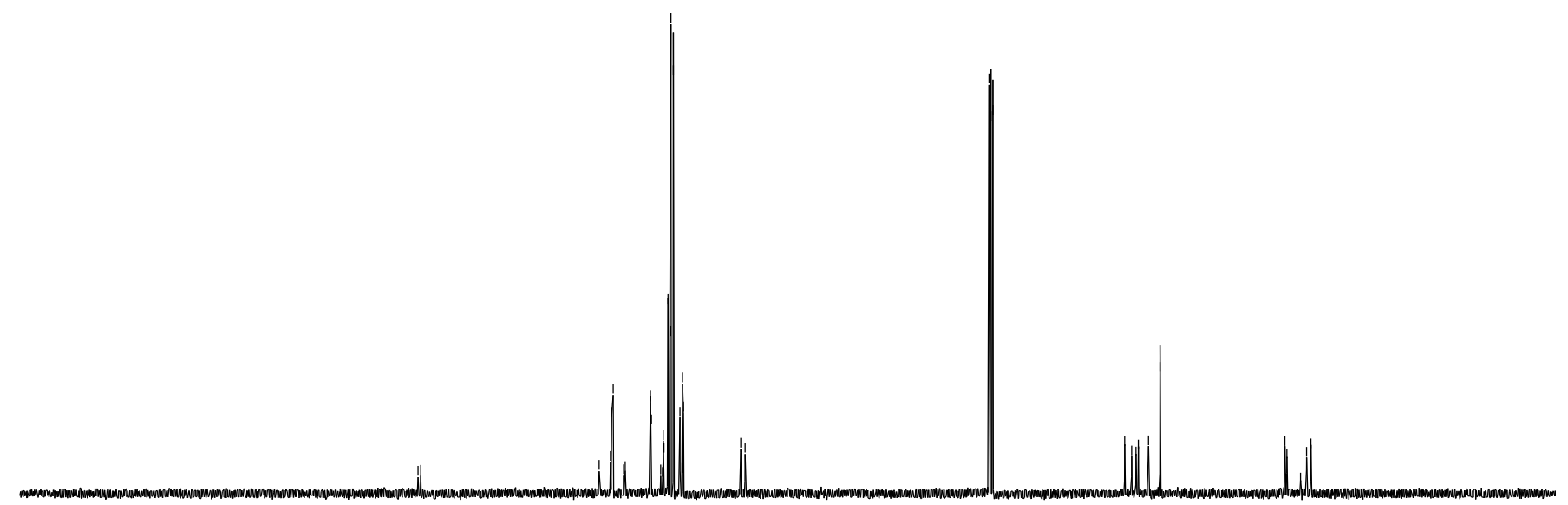




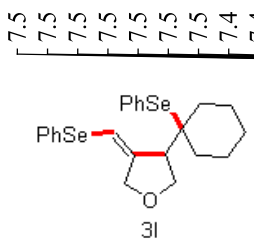

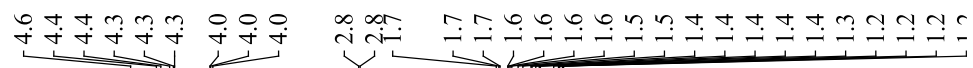

${ }^{1} \mathrm{H} \mathrm{NMR}\left(400 \mathrm{MHz}, \mathrm{CDCl}_{3}\right)$

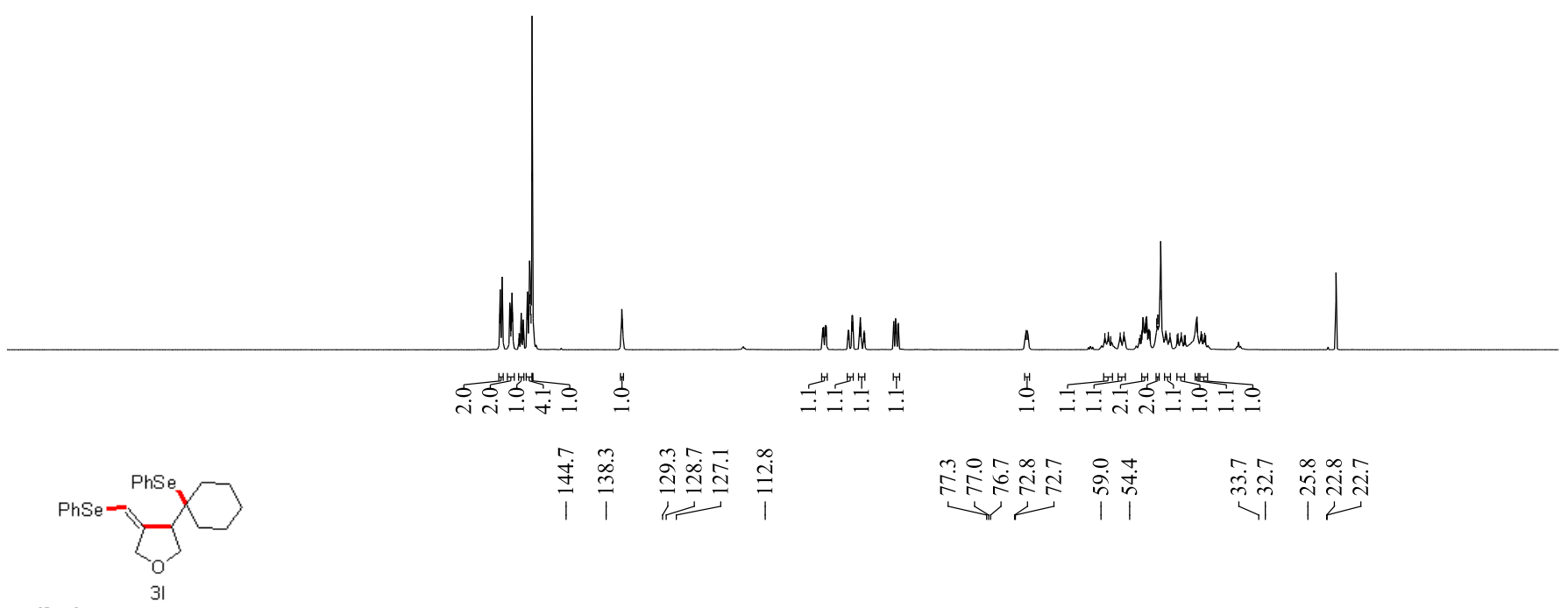

${ }^{13} \mathrm{C}\left\{{ }^{1} \mathrm{H}\right\} \operatorname{NMR}\left(101 \mathrm{MHz}, \mathrm{CDCl}_{3}\right)$

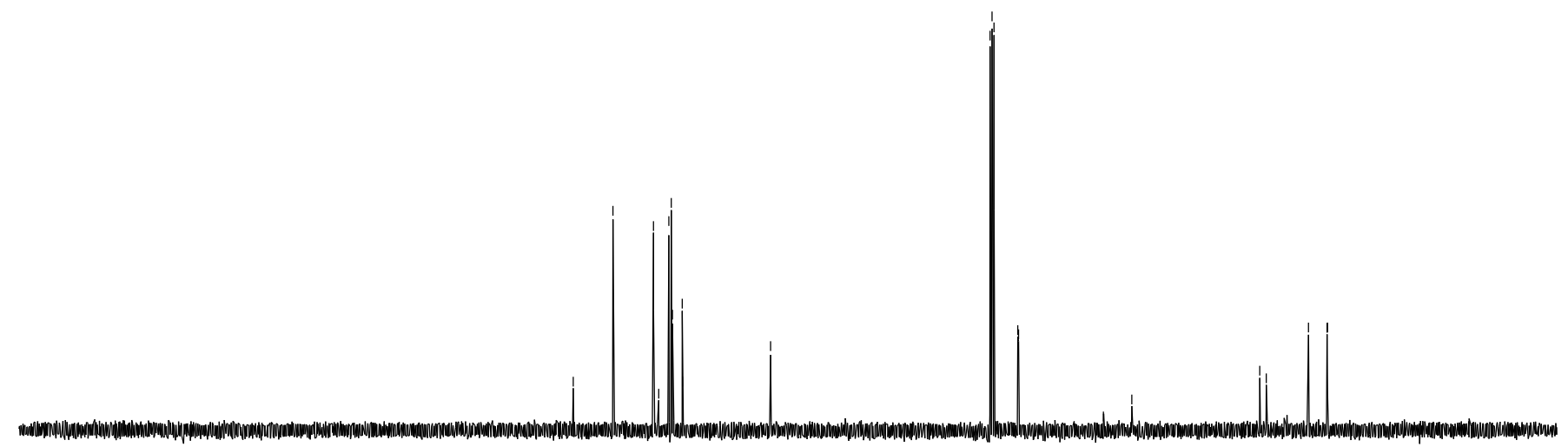




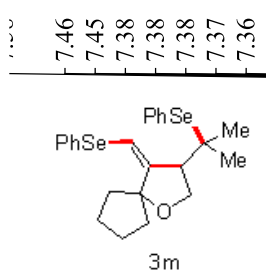

${ }_{1} \mathrm{H} \mathrm{NMR}(400 \mathrm{MHz}, \mathrm{CDC} 1)$

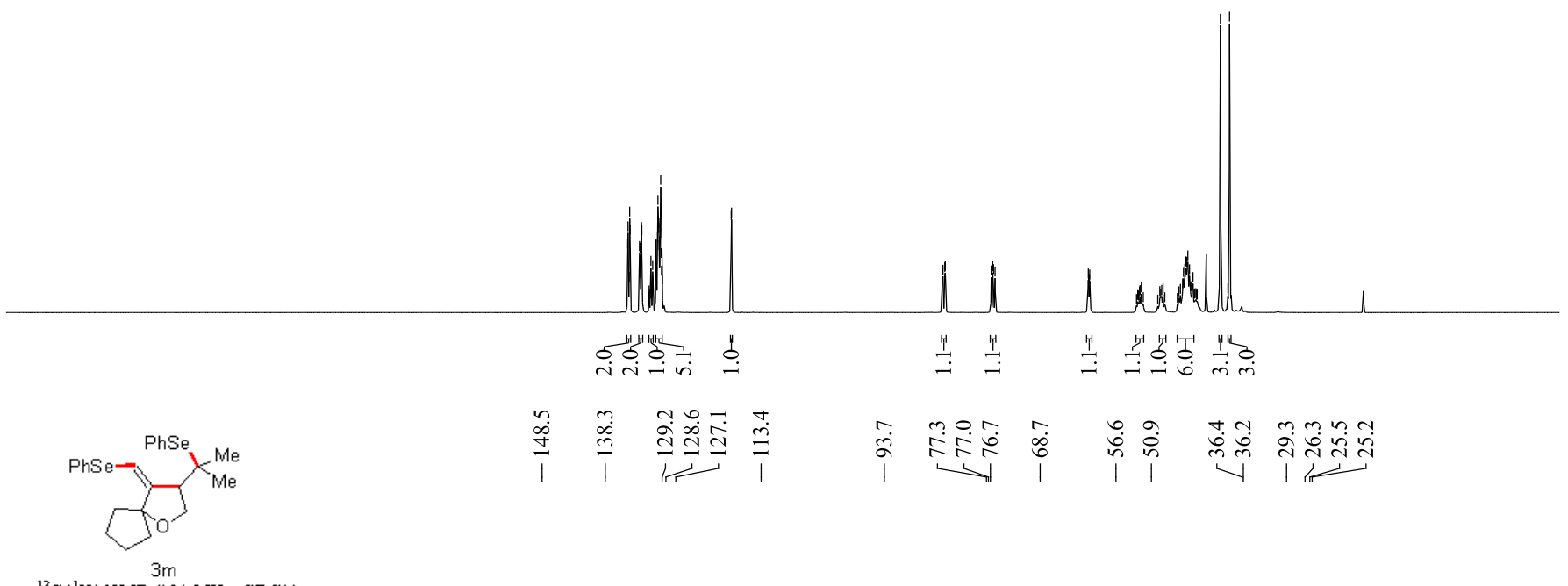

${ }^{13} \mathrm{C}\left\{{ }^{1} \mathrm{H}\right\} \mathrm{NMR}\left(101 \mathrm{MHz}, \mathrm{CDCl}_{3}\right)$

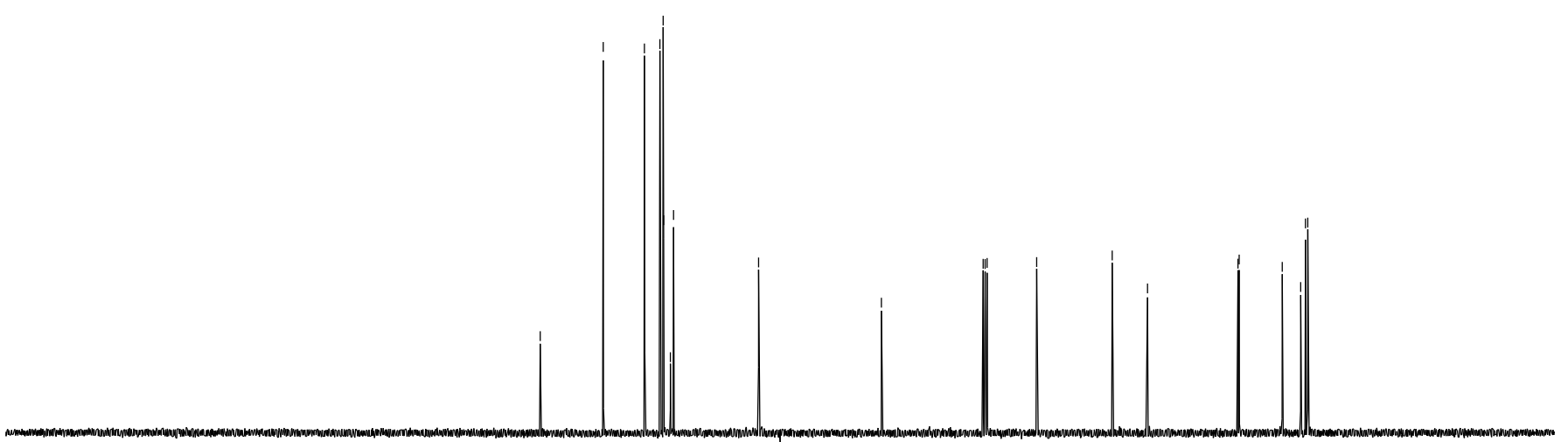




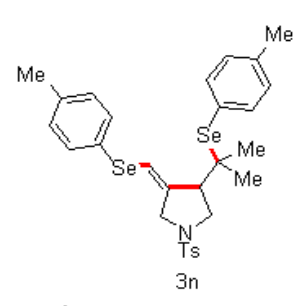

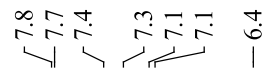

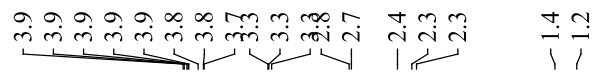

${ }^{1} \mathrm{H} \mathrm{NMR}\left(400 \mathrm{MHz}, \mathrm{CDCl}_{3}\right)$
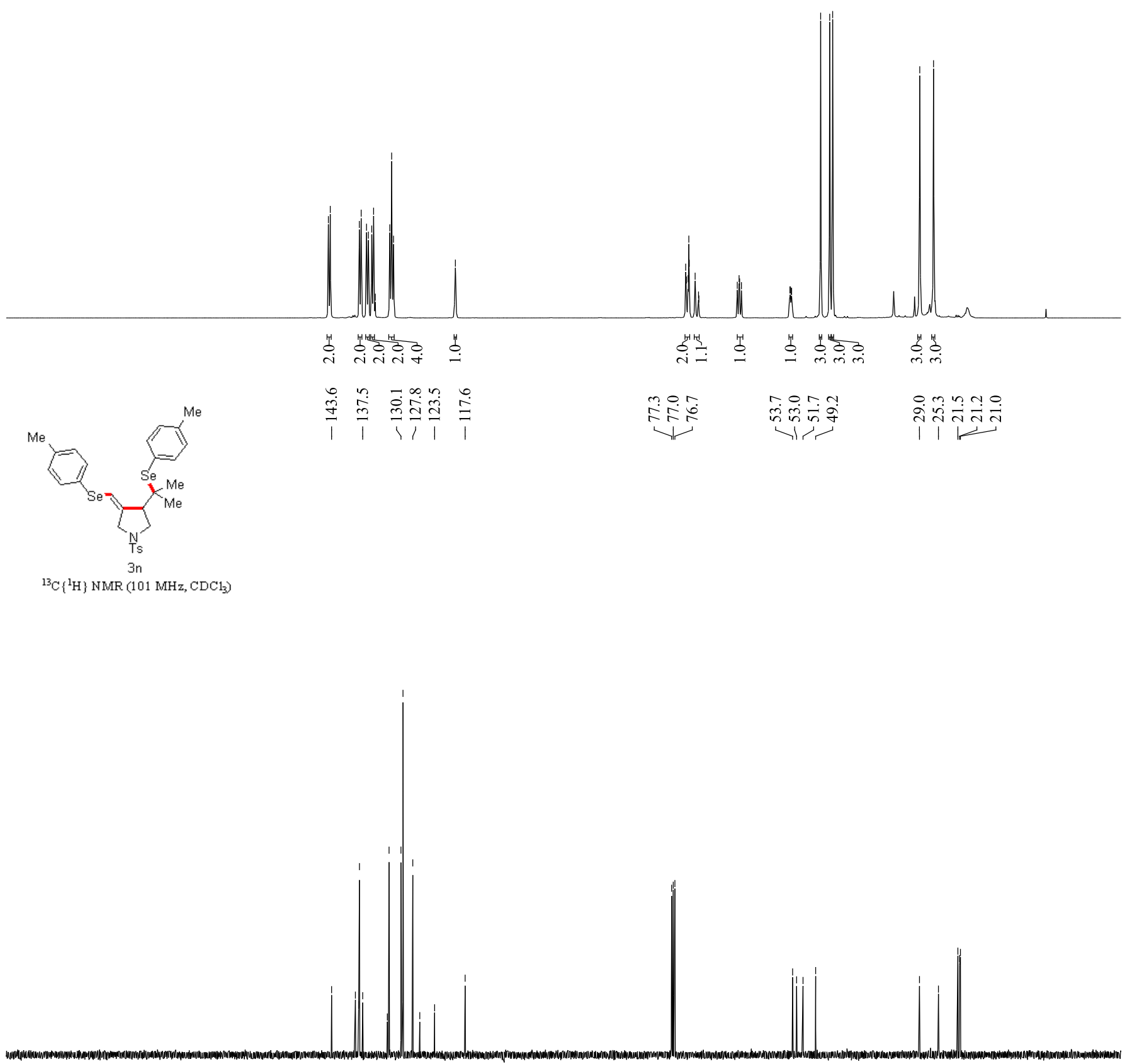


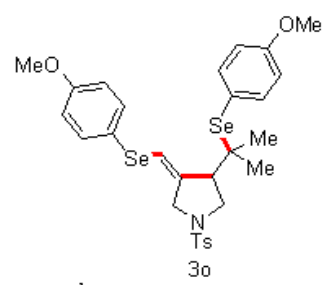

$$
\text { irị }
$$

${ }^{1} \mathrm{H} \mathrm{NMR}\left(400 \mathrm{MHz}, \mathrm{CDCl}_{3}\right)$
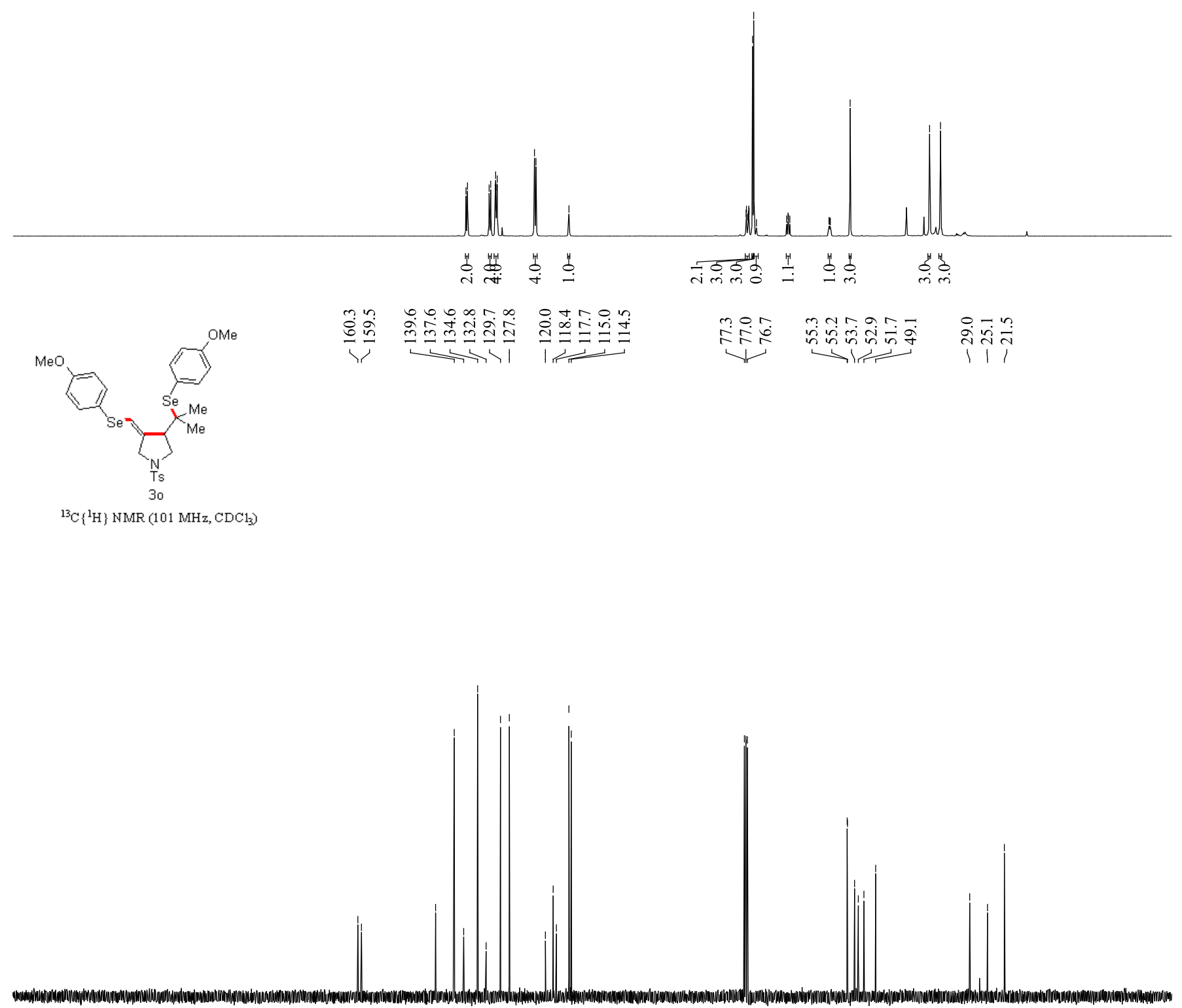


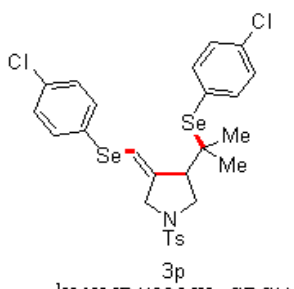

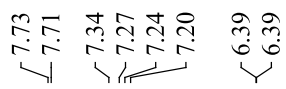

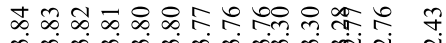

กํฺุุ
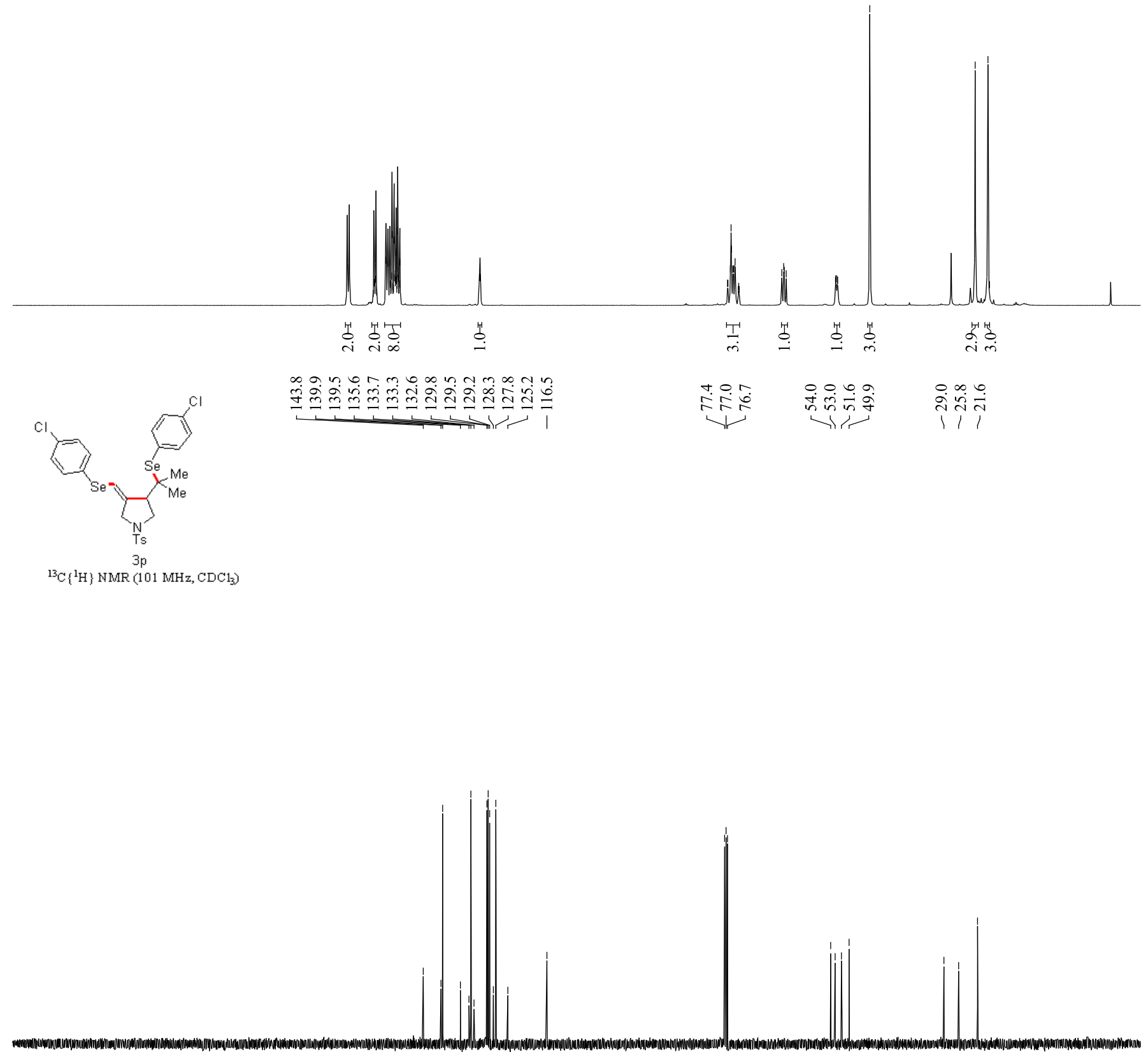

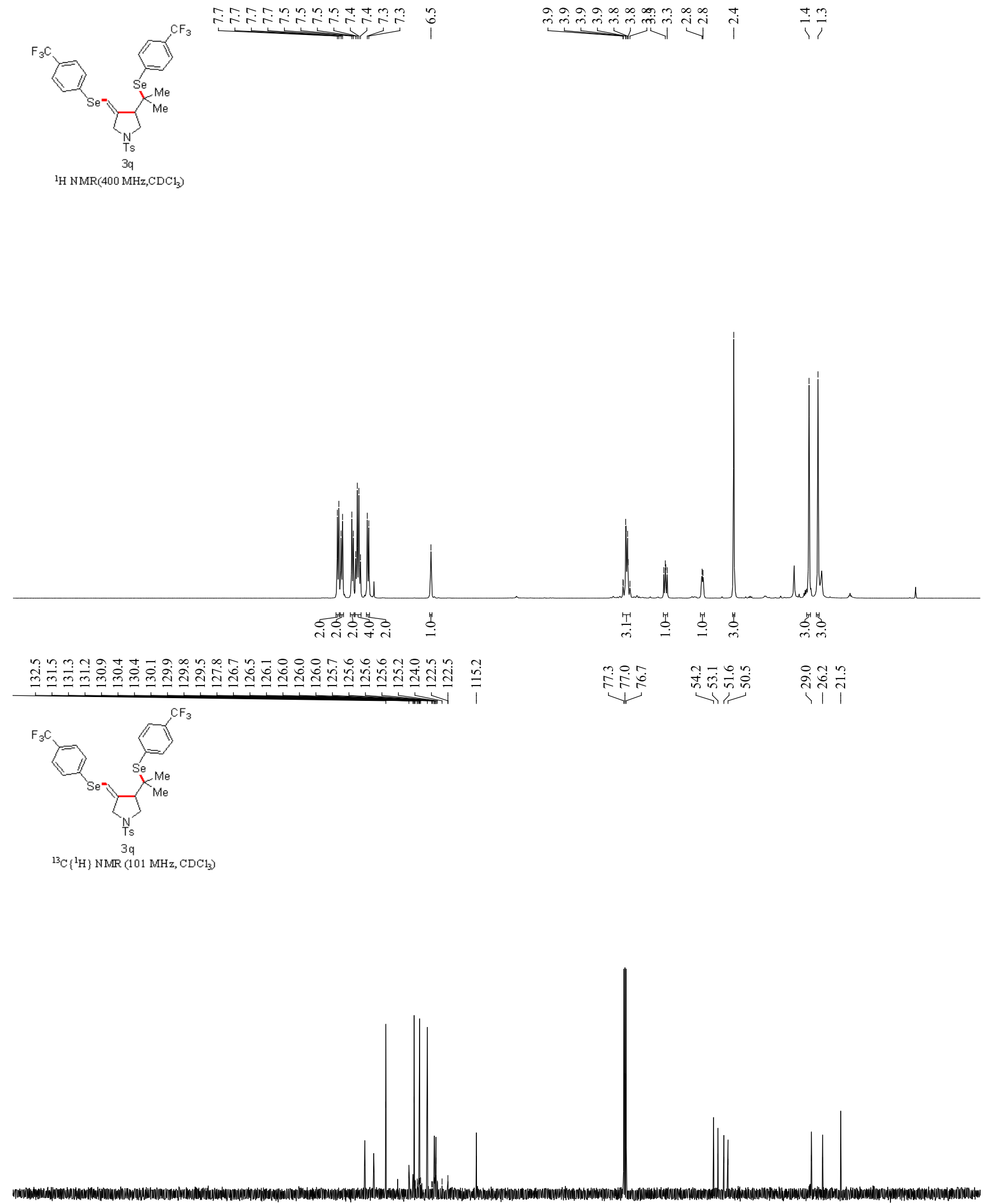

$\begin{array}{llllllllllllllllllllllllllllll}230 & 220 & 210 & 200 & 190 & 180 & 170 & 160 & 150 & 140 & 130 & 120 & 110 & 100 & 90 & 80 & 70 & 60 & 50 & 40 & 30 & 20 & 10 & 0 & -10\end{array}$ 


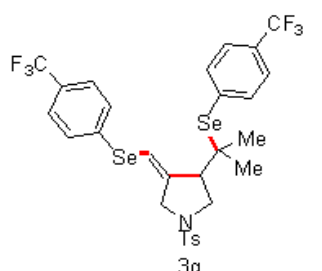

${ }^{19} \mathrm{~F}\left\{{ }^{1} \mathrm{H}\right\} \operatorname{NMR}\left(376 \mathrm{MHz}, \mathrm{CDCl}_{3}\right)$

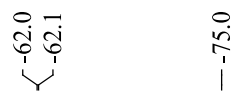
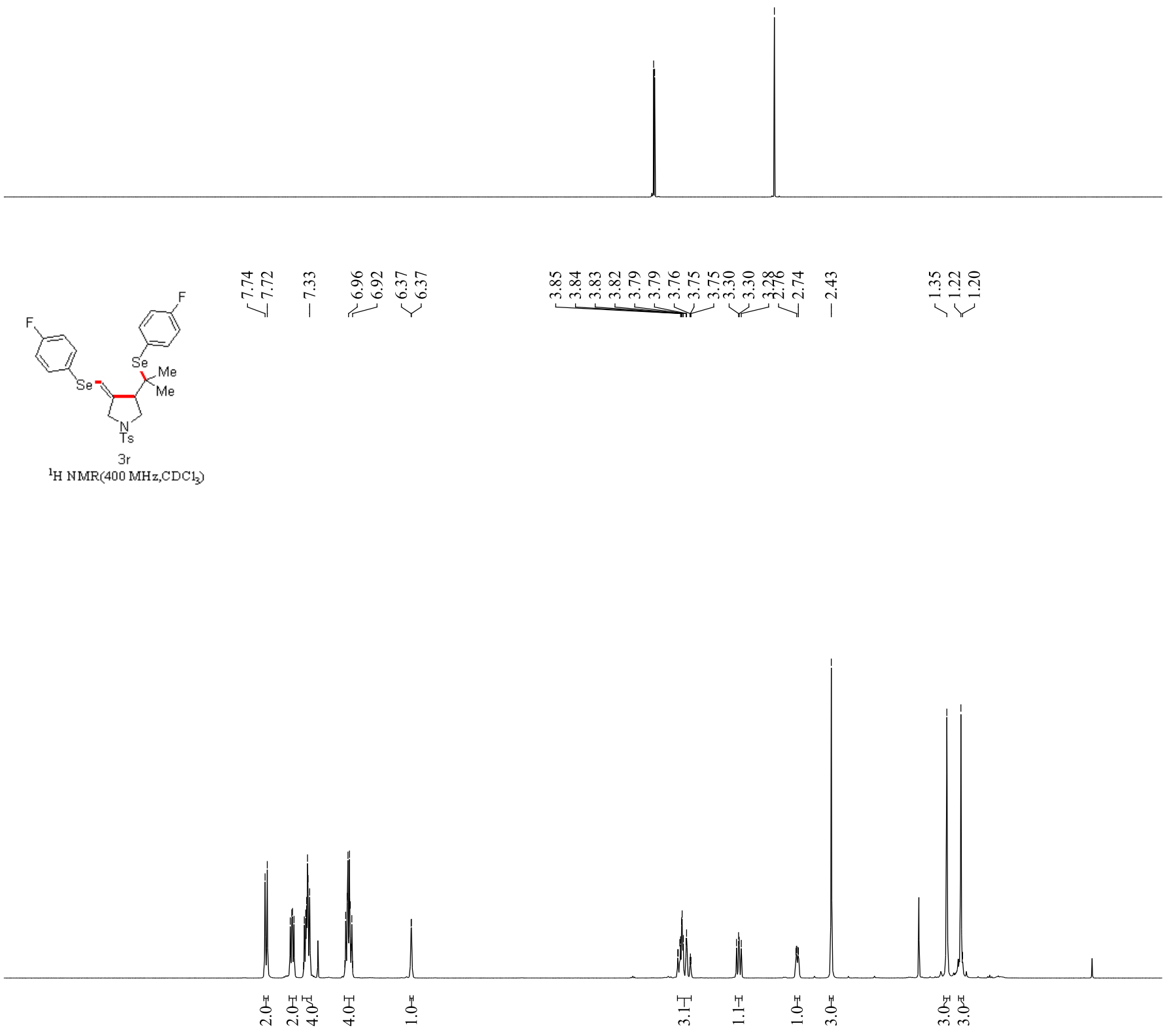


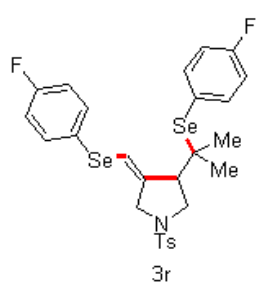

${ }^{13} \mathrm{C}\left\{{ }^{1} \mathrm{H}\right\}$ NMR $\left(101 \mathrm{MHz}, \mathrm{CDCl}_{3}\right)$

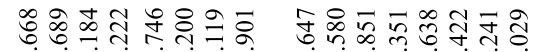

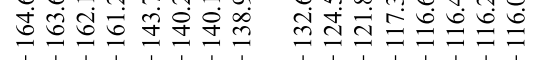

$\stackrel{5}{2}$

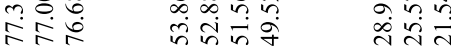

年 ज行
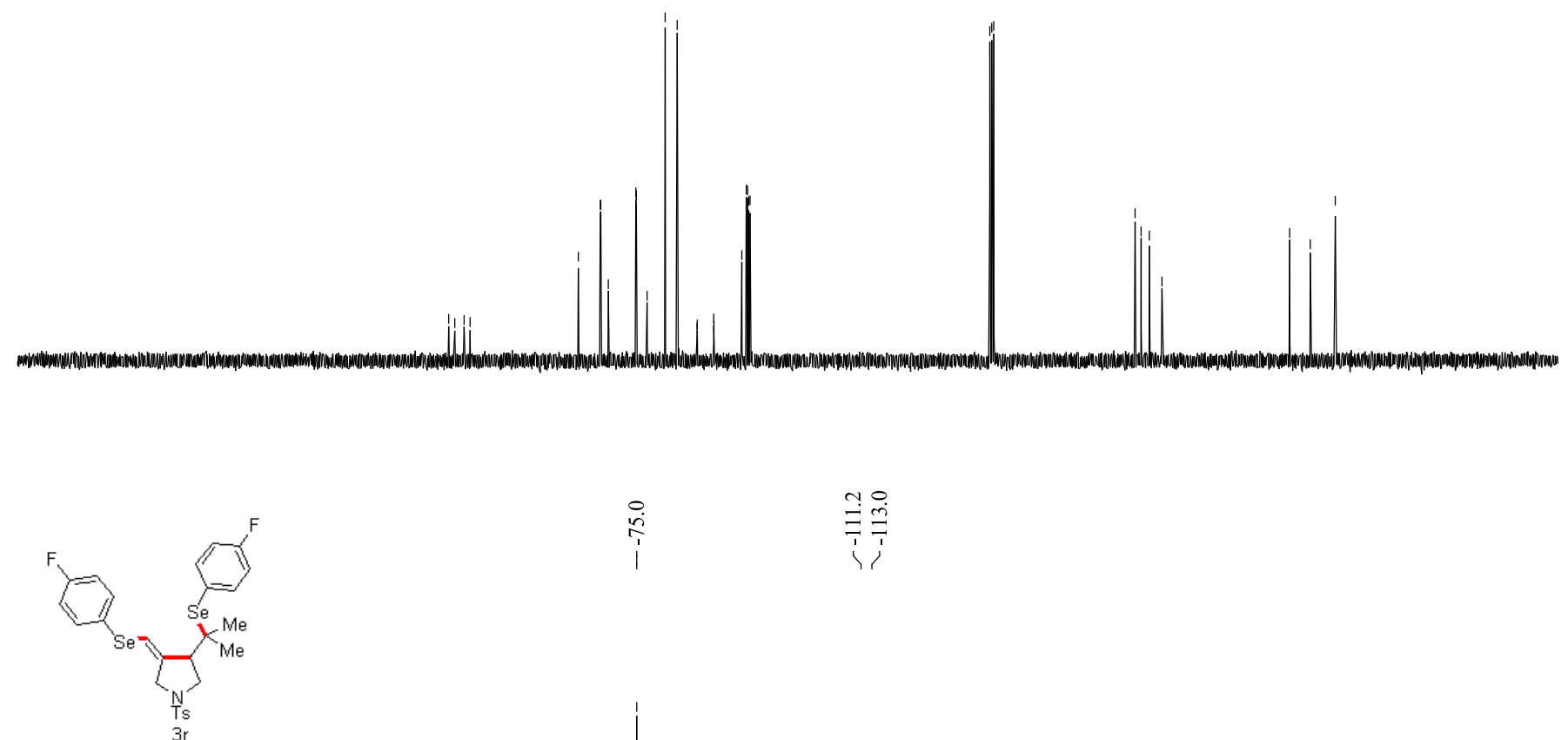

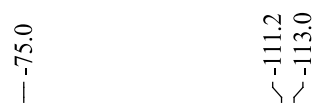

${ }^{19} \mathrm{~F}\left\{{ }^{1} \mathrm{H}\right\} \operatorname{NMR}\left(376 \mathrm{MHz}, \mathrm{CDCl}_{3}\right)$ 


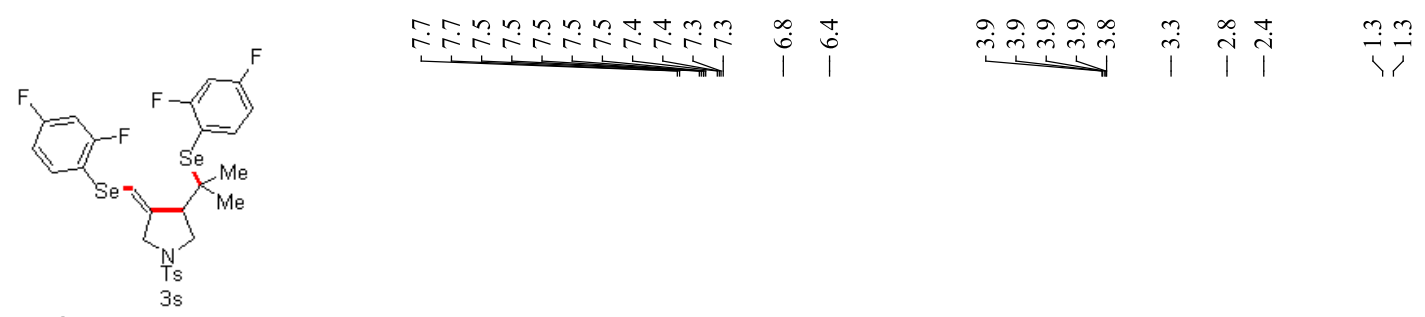

${ }^{1} \mathrm{H} \mathrm{NMR}\left(400 \mathrm{MHz}, \mathrm{CDCl}_{3}\right)$

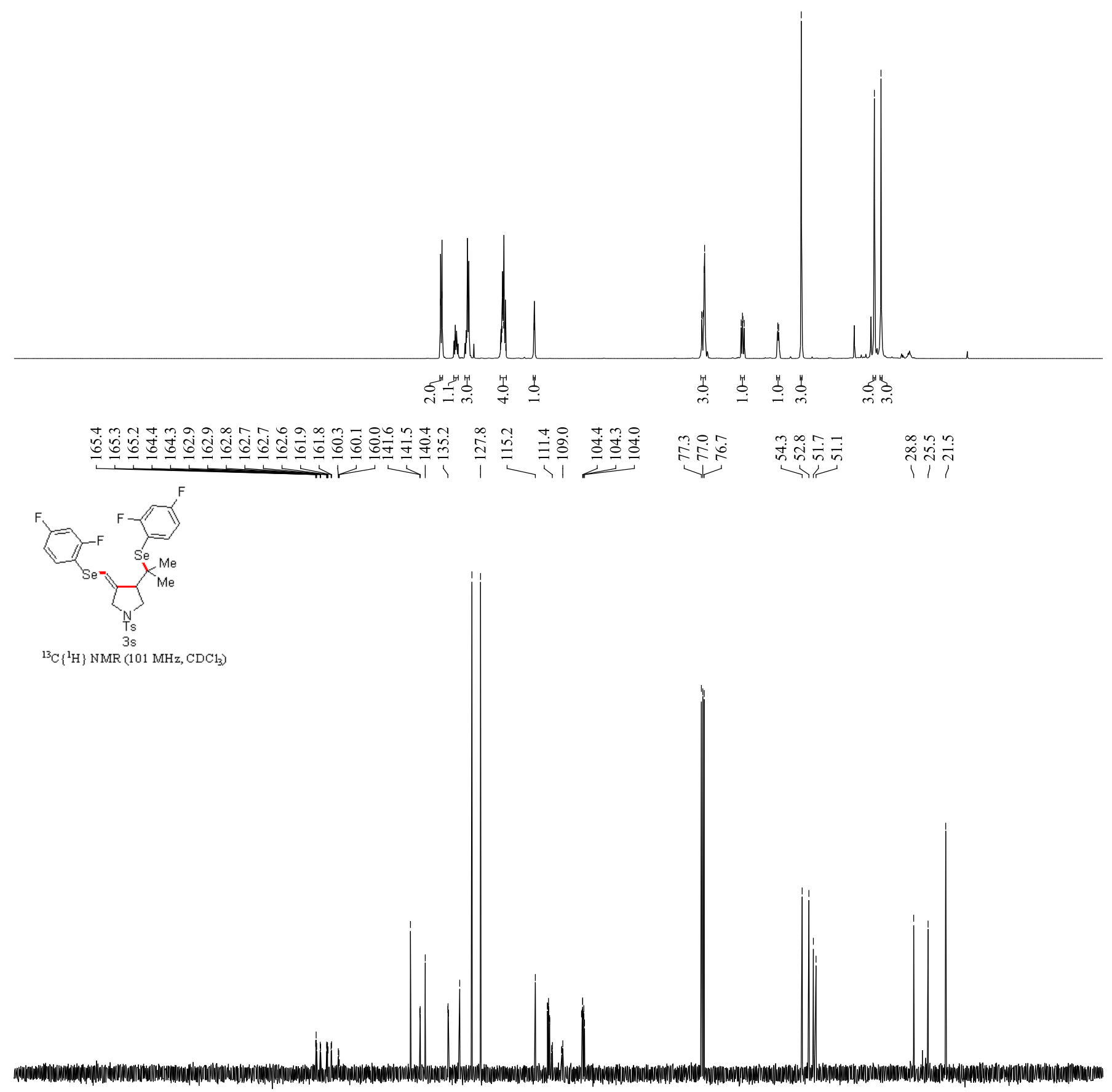




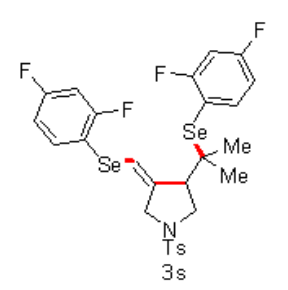

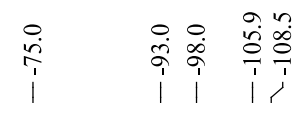

${ }^{19} \mathrm{~F}\left\{{ }^{1} \mathrm{H}\right\}$ NMR $\left(376 \mathrm{MHz}, \mathrm{CDCl}_{3}\right)$

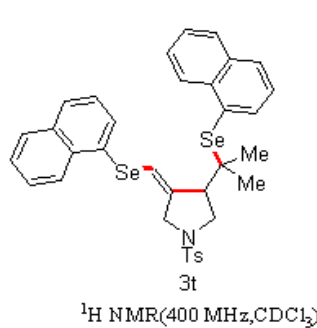

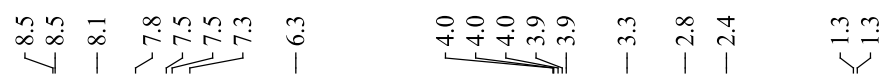

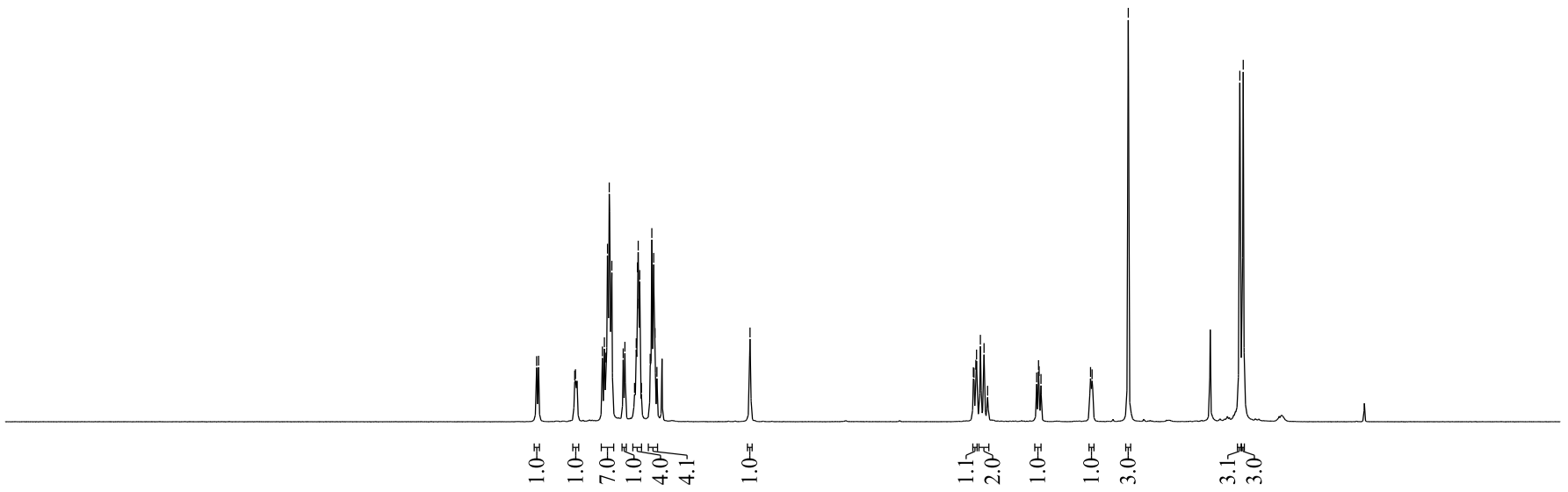



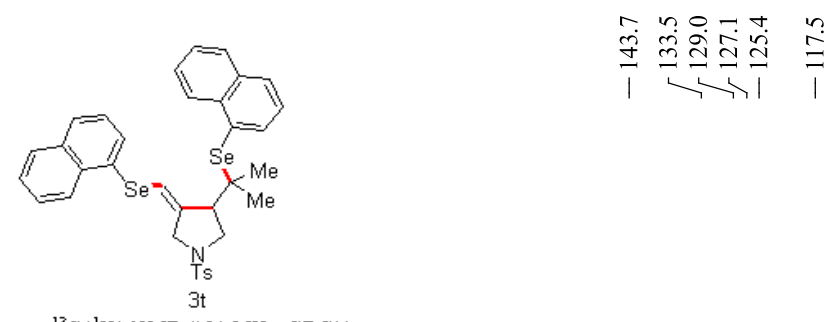

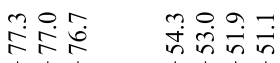

垈

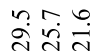

१र्त

${ }^{13} \mathrm{C}\left\{{ }^{1} \mathrm{H}\right\} \operatorname{NMR}\left(101 \mathrm{MHz}, \mathrm{CDCl}_{3}\right)$

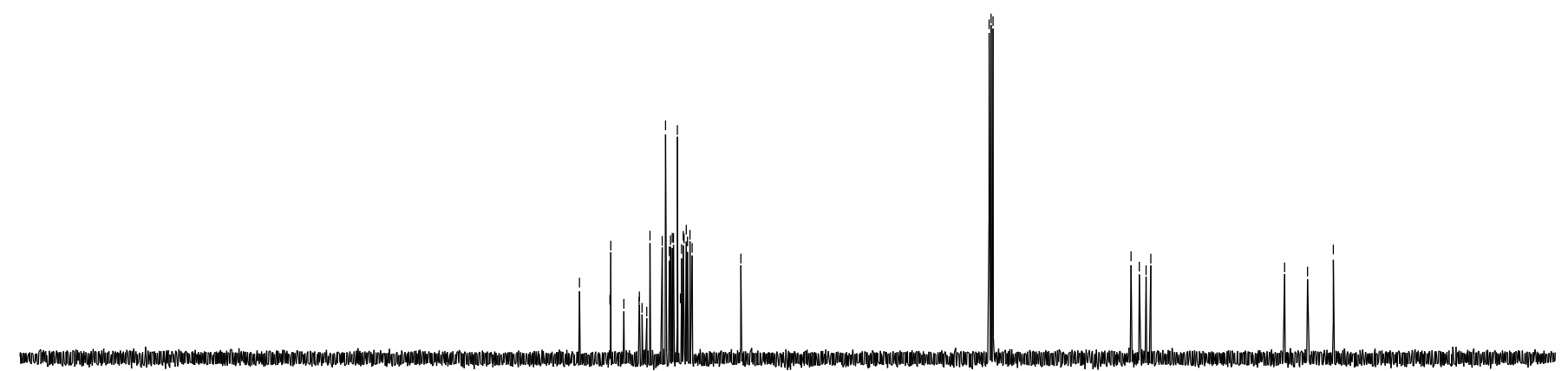

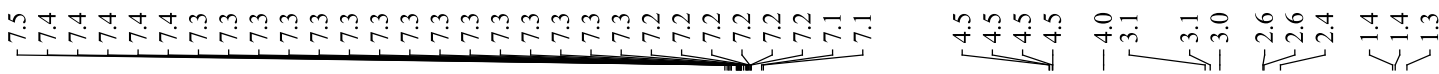

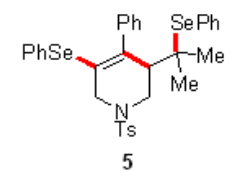

${ }^{1} \mathrm{H} \mathrm{NMR}_{\left(400 \mathrm{MHz}, \mathrm{CDCl}_{3}\right)}$

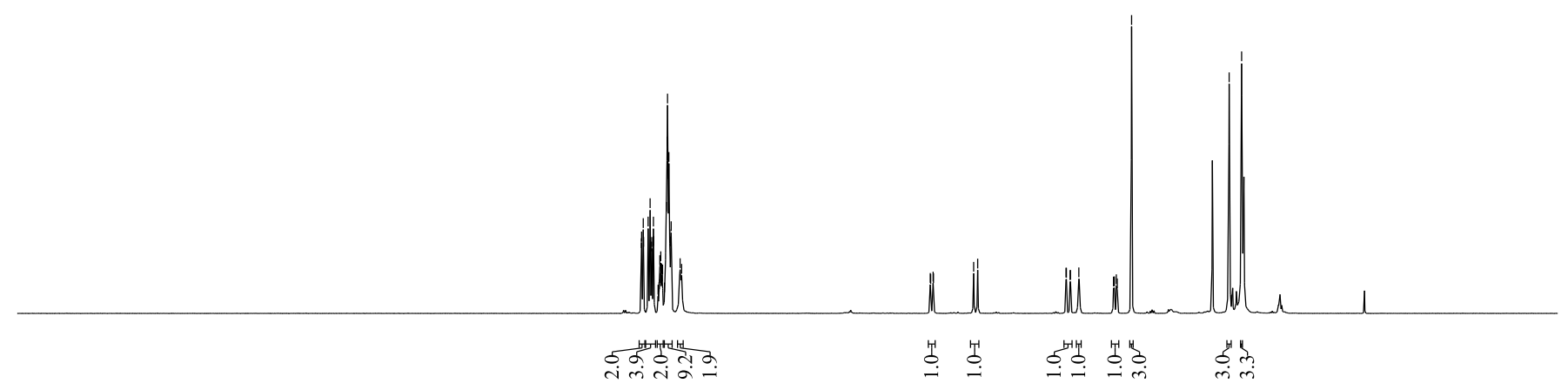




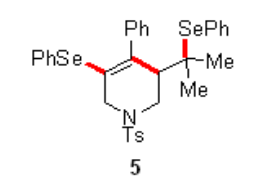

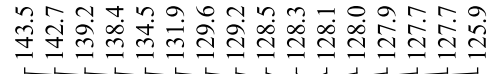

${ }^{13} \mathrm{C}\left\{{ }^{1} \mathrm{H}\right\} \mathrm{NMR}\left(101 \mathrm{MHz}, \mathrm{CDCl}_{3}\right)$
诲 谣

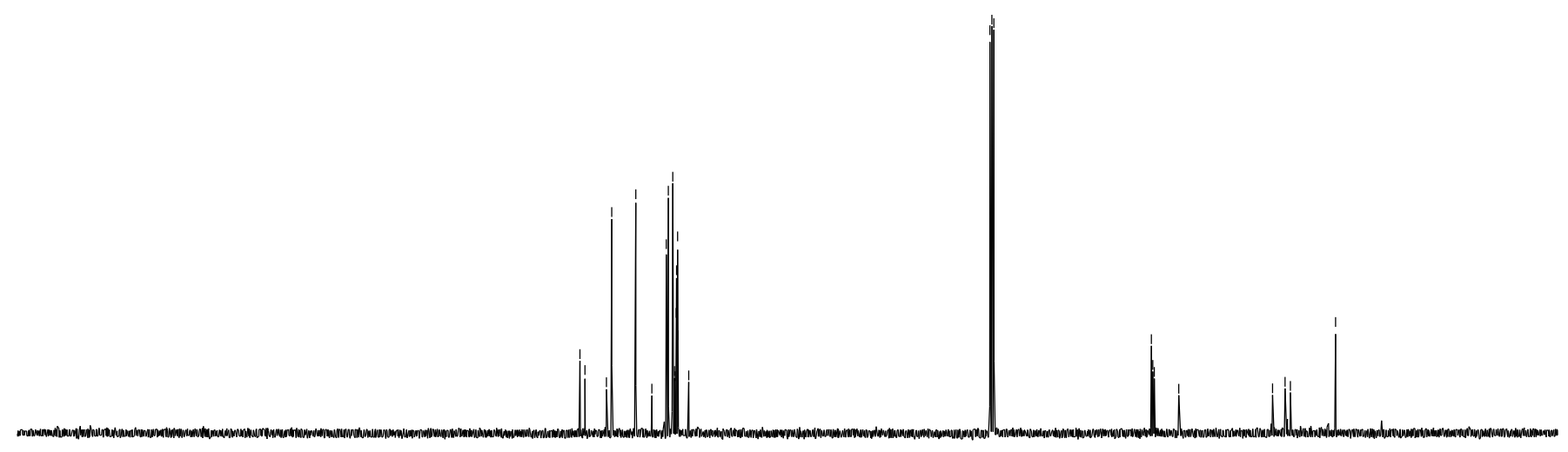

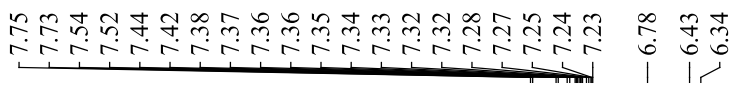

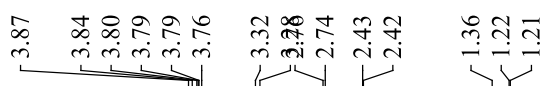

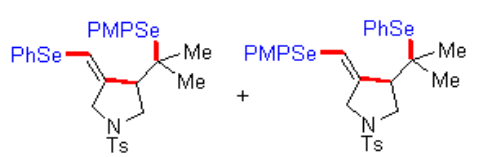

$\left.{ }^{1} \mathrm{H} \mathrm{NMR}_{(400 \mathrm{MHz}, \mathrm{CDC1}}\right)$

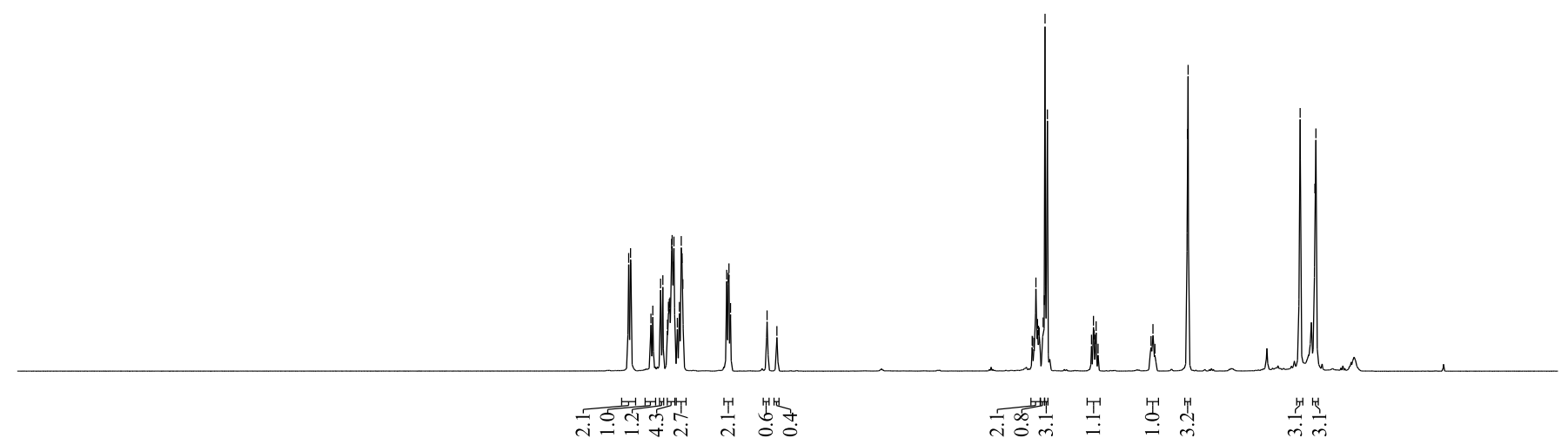




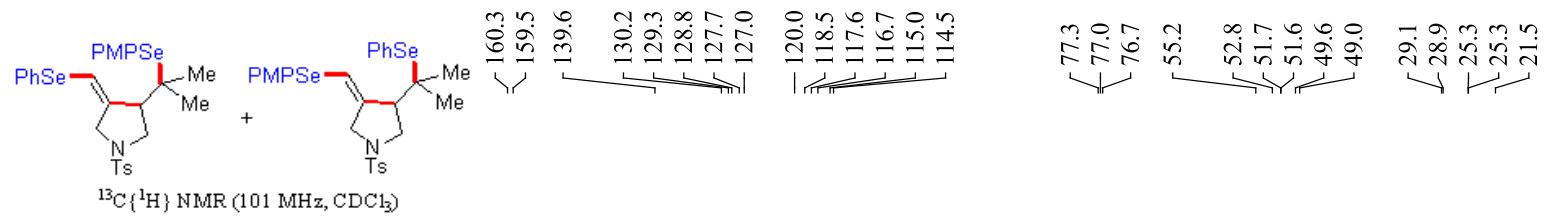

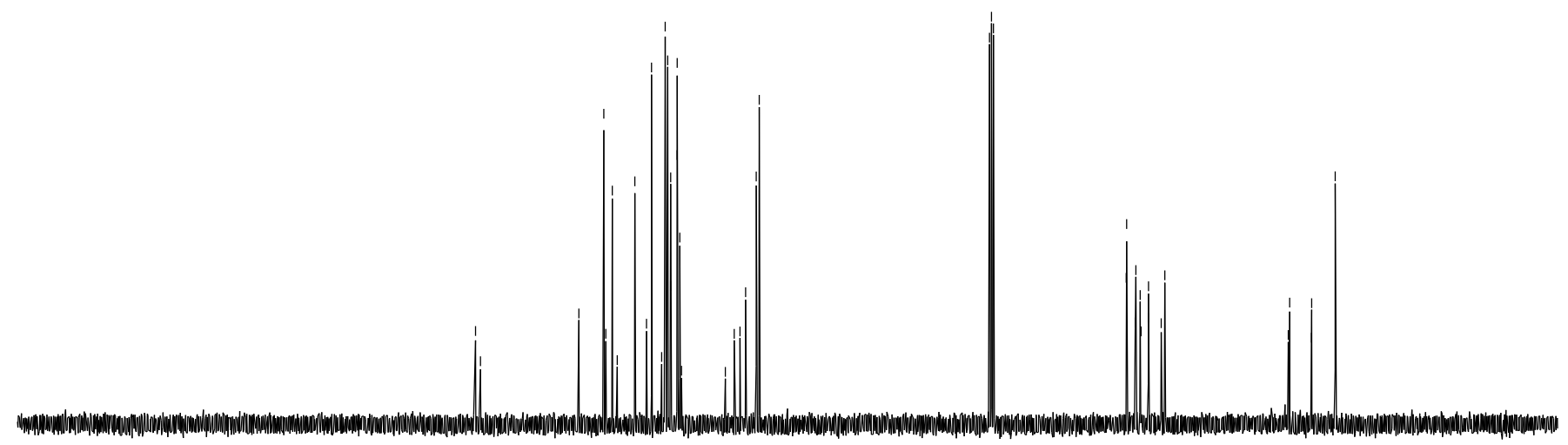

